\title{
Conformational behavior of stereo regular substituted polyglycolides is side chain dependent
}

\author{
Fateh S. Nandel ${ }^{*}$, Roobee Garla
}

Department of Biophysics, Panjab University, Chandigarh, India; ${ }^{*}$ Corresponding Author: fateh nandel@yahoo.com

Received 8 April 2011; revised 13 May 2011; accepted 17 May 2011.

\begin{abstract}
Substituted polyglycolides having two asymmetric centers are attractive alternatives to materials derived from petroleum because of their biocompatibility and biodegradability. The conformational behavior of various substituted polyglycolides has been investigated by both quantum mechanical and molecular dynamics approaches. Polymethylglycolide (polylactide) and polyphenylmethylglycolide in RS or SR forms are predicted to adopt $2_{7}$ ribbon type structures with $\varphi, \Psi$ values of $\pm 30, \pm 50$ or $\mp 30$ or $\mp 50$ respectively stabilised by carbonyl-carbonyl interactions. Isopropylglycolide and isobutylglycolide having branching at $\beta$ \& $\gamma$ positions respectively in their side chains can be realized in all SS form with $\varphi, \Psi$ values lying in right handed helical region. In addition to carbonylcarbonyl interactions, the hydrophobic interactions between the side chains in isopropylglycolide the $\mathrm{C}-\mathrm{H}-\mathrm{O}$ interactions also contributes to the stability. With cyclic side chains directly attached to $\mathrm{C}_{\alpha}$ of backbone, polyphenylglycolide (polymandelide) and polycyclohexylglycolide are found to adopt left handed helical structure without hydrogen bonds in RR form, stabilised by stacking interactions and hydrophobic interactions respectively. In all the forms of polyphenylglycolide \& polycyclohexylglycolide, the cyclic side chains are found to be locked into unfavourable gauche plus conformation. The stability of substituted polyglycolides has been analyzed in terms of various interactions. The carbonyl-carbonyl interactions in all the conformations of all forms of substituted polyglycolides are found to be of highly shielded parallel motif with only one short carbon-oxygen interaction. Simulation studies of substituted polyglycolides in water give a good insight of the approach of water molecules to the
\end{abstract}

backbone.

Keywords: Polyglycolides; Conformation; 27 Ribbon Type Structure; Helical Structure without Hydrogen Bonds; Carbonyl-Carbonyl Interactions; Simulation Studies

\section{INTRODUCTION}

Conventional polymers such as polyethylene, polypropylene and polystyrene persist for many years after disposal due to their resistance to degradation which creates serious environmental problems. This has lead to the search of biodegradable polymers which degrade by the action of microorganisms such as bacteria, fungi and may also be broken down by non-enzymatic processes such as chemical hydrolysis and the degraded products should be non-toxic to living organisms directly or indirectly. Polylactides/polymethylglycolide (PLA), as one of commercially available and biomass-derived polymers, has been attracting much attention from both academic and industrial view points. It has tremendous potential in both traditional and non-traditional applications where thermoplastics are employed. It is because of these reasons, polylactide (PLA) has been explored to a fairly good extent for medical applications [1-16] as rod, plate, screw, fibre, sheet, sponge, beads for bone and tissue engineering [17-20], microsphere for drug delivery system [21] films and foils for wound treatment. PLA have also found applications in agriculture as mulch films and for slow release of pesticides and fertilisers. PLA is widely used in packaging for a broader array of products [22-27] because the level of lactic acid that migrates to food from packaging containers is much lower than the amount of LA used in common food ingredients [28]. Li et al. have recently reported the chromatographic separation of polylactides of different stereo chemical compositions on the basis of their solubility in THF [29]. Further, modern and emerging production technologies have lowered its production costs [22-23, 30-31] as it can be now obtained from a renewable 
source (corn) with high yield.

Poly-lactic-acid (PLA) has been investigated mainly in the all S form-PLLA [32-35] and there are few reports dealing with poly-D-lactic-acid-PDLA [35-36]. Depending on the preparative conditions, PLLA crystallizes in three forms i.e. $\alpha, \beta$ and $\gamma$ and PLLA is reported to adopt right handed helical structure. Theoretical calculations on the conformational behaviour of PLLA have been carried out in terms of rotational isomeric state model and Kang et al. have analyzed the vibrational spectra of PLLA in the light of the results of this model [37]. Meaurio et al. had studied the distribution of conformer population for the semi-crystalline and amorphous PLLA by curve fitting the experimentally attained FTIR spectra. [38] Pan et al. [39] have demonstrated that the glass transition temperature of PLLA can be determined from temperature dependent FTIR intensity of $1267 \mathrm{~cm}^{-1}$ band arising due to $v_{\text {as }}(\mathrm{C}-\mathrm{O}-\mathrm{C})+\delta(\mathrm{CH})$ vibrational mode. The substituted diglycolides [Ac$\left.\left\{\mathrm{O}-\mathrm{C}^{*} \mathrm{H}(\mathrm{X})-\mathrm{CO}\right\}_{2}-\mathrm{OCH}_{3}\right]$ contain two chiral centres giving rise to identical RR/SS or enantiomeric $R, S / S, R$ forms. Polyglycolides with different alkyl or cyclic (phenyl \& cyclohexyl) side chains have been studied with emphasis only on the glass transition temperature using different techniques like DSC, NMR etc. [40-43]. Thus, the conformational behaviour of substituted polyglycolides, in all the forms in the glass transition temperature region remains unexplored.

The backbone of substituted polyglycolides is structurally similar to that of peptide back bone in proteins as the ester bond is isoelectronic to peptide bond. Thus, polylactides/substituted polyglycolides lack amide hydrogen and precluding the formation of hydrogen bond networks that stabilize the peptides secondary structure like helices and beta sheets. A systematic study of the conformational behaviour of various substituted polyglycolides i.e. polymethylglycolide, polyphenylmethylglycolide, isopropylglycolide, isobutylglycolide polyphenylglycolide \& polycyclohexylglycolide in all RR, all SS and enatiomeric R, S and S, R forms as a function of chain length is worth investigating from the viewpoints: 1) do the polyglycolides adopt secondary structure like peptides or not; 2) do the side chains influence population of a particular form; and 3) what type of interactions stabilizing the adopted structure.

The use of polymers for various applications is characterized by the glass transition temperature [44] which in turn depends upon the nature and type of structure adopted by these polymers and what type of interactions stabilize the adopted conformations. The analysis of the interactions in the most stable states of various forms may provide insight into the nature of glass transition temperature. Knowledge of the interactions may aid in designing of polymers with desired $\mathrm{T}_{\mathrm{g}}$ value by incorporating stretches of polymer's and that too in desired forms i.e. all $\mathrm{R}$, all $\mathrm{S}$, alternate $\mathrm{R}, \mathrm{S}$ forms etc. The simulation studies of substituted polyglycolides in different forms carried out in water, will throw light on the approach of water molecules to the ester moiety and hence, about their degradability.

\section{METHODOLOGY}

The backbone of substituted polyglycolides shown in Figure 1 is similar to peptide backbone. Therefore, the torsion angles have been defined in a similar manner. The bond lengths and bond angles were taken from the literature [45]. The ester group $\mathrm{C}_{\alpha} \mathrm{COOC}_{\alpha}$ in its trans configuration was assumed to be planar. The torsion angles were varied systematically, initially in steps of $30^{\circ}$. Energy calculations were carried out using the quantum mechanical method PCILO [46] (perturbative configuration interaction of localized orbital) on a Sun W, Ultra 5-10; Sparc workstation. It may be mentioned that the minima obtained by PCILO calculations were also the minima at the $a b$ initio level for the usual amino acids, N-methylated and dehydro amino acids. In addition, the PCILO results $[47,48]$ for peptides containing usual [49] and unusual amino acids [50-51] were in conformity with $a b$ initio results [52,53] and knowledge-based crystallographic data. [54,55] The global, local, and low-energy minima $\&$ their combinations obtained in the $\varphi, \psi$ maps and $\chi$ curves/maps in substituted diglycolides of the form Ac- $\left\{\mathrm{O}-\mathrm{C}^{*} \mathrm{H}(\mathrm{X})-\mathrm{CO}\right\}_{2}-\mathrm{OCH}_{3}$ in identical \& enantiomeric forms, have been used to generate the conformational states for substituted polyglycolides. The energies of all the states thus generated have been calculated. The single point energy calculations for $30^{\circ}$ interval was further refined by varying $\varphi, \psi$ and $\chi$ values in the neighbourhood of the minima, in steps of $5^{\circ}$ and then, in steps of $2^{\circ}$.

The substituted polyglycolides are used for different applications and in different environment therefore, Molecular Dynamic (MD) simulation study in water has been carried out with the help of GROMACS software [56] on the desktop computer, dual Intel Pentium 4, 3.0 $\mathrm{GHz}$. It is worth mentioning that the simulation results obtained by GROMOS force field are found to be in good agreement with the experiment for peptides \& peptoids [57]. The starting geometry for simulation studies

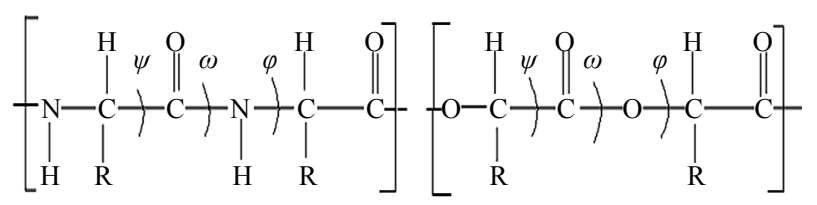

Figure 1. Similarity between peptide and glycolide backbone. 
has been taken from QM results for all these molecules. The Dundee-PRODRG2 [58] server was used to obtain the GROMACS topology and coordinate files. Interaction parameters within the design sequence were taken from GROMOS-96 force field ffgmx. Energy of the system was minimized by the Steepest Descent Method, using the convergence criteria of $50 \mathrm{~kJ} \cdot \mathrm{mol}^{-1}$ followed by Conjugate Gradient method with a force constant of $20 \mathrm{~kJ} \cdot \mathrm{mol}^{-1}$. Next, the MD run was carried out in vacuum for $2 \mathrm{~ns}$, with a time step of 2 ps using the Leap Frog Algorithm [59]. The temperature was controlled through weak coupling to a bath of constant temperature [60] using a coupling time; $\tau_{\mathrm{p}}$ of $0.1 \mathrm{ps}$ and a reference temperature; $\mathrm{T}_{0}$ of $300 \mathrm{~K}$. The LINCS algorithm [61] was used to restrict all bonds to their equilibrium lengths and the center of mass motion of the system was removed every step to maintain the effective simulation temperature at $300 \mathrm{~K}$. For the evaluation of coulomb interactions and van der Waals interaction a cut off of 0.9 and $1.0 \mathrm{~nm}$ respectively was applied. Long range forces were updated every $10 \mathrm{fs}$ during generation of the neighbor list. The Long Range Electrostatic Interactions were calculated using a Particle Mesh Ewald Summation [62]. Initial velocities of all atoms were obtained from a Maxwellian distribution at the desired initial temperature. On the basis of the final conformation obtained for polyglycolides after 2 ns simulation in vacuum MD, a simple cubic periodic box was set up using the Simple Point Charge (SPC) Water Model [63]. For equilibration of solvent around the model polymers under study, their position was restrained for 20 ps and finally, the MD simulation of $1 \mathrm{~ns}$ at $300 \mathrm{~K}$, were performed without any restrain. The pressure was controlled using weak coupling with a time constant of 0.5 ps and a reference pressure of 1 Bar.

\section{RESULTS AND DISCUSSION}

In the beginning, the conformational behaviour of the substituted diglycolides of the type Ac- $\left\{\mathrm{O}-\mathrm{C}^{*} \mathrm{H}(\mathrm{X})\right.$ $\mathrm{CO}\}_{2}-\mathrm{OCH}_{3}$ (with $\mathrm{X}$ being methyl, alternate phenyl \& methyl, isopropyl, isobutyl, phenyl and cyclohexyl) having two chiral centres have been investigated in different forms, i.e. all RR, all $\mathrm{SS}$, enantiomeric $\mathrm{R}, \mathrm{S} / \mathrm{S}, \mathrm{R}$ etc. and the results are summarised in Table 1 . The results have further been optimised up to $2^{\circ}$ for all the states in different forms and only the results for most stable states of all forms are tabulated in Table 2 . It is obvious from the results that the predicted states both for Ac- $\left\{\mathrm{O}-\mathrm{C}^{*} \mathrm{H}\left(\mathrm{CH}_{3}\right)-\mathrm{CO}\right\}_{2}-\mathrm{OCH}_{3} \&$ Ac-(phenylmethylgycolide) $-\mathrm{OCH}_{3}$ lay within $2 \mathrm{kcal} \cdot \mathrm{mol}^{-1}$, thus, these molecules can be realized in all the above forms. The enantiomeric $\mathrm{R}, \mathrm{S} / \mathrm{S}, \mathrm{R}$ forms in both molecules are found to be degenerate with $\varphi, \psi$ values of $\pm 30^{\circ}, \pm 50^{\circ}$ or $\mp 30^{\circ}$ or $\mp 50^{\circ}$ respectively. The $\varphi, \psi$ values for low energy states in Table 2 for all the forms, also lie in the neighborhood of the $\varphi, \psi$ values for the most stable states. The results obtained for lactide are somewhat at variance with reported results in the SS form [64]. Isopropylglycolide and isobutylglycolide, with branching in the side chain at $\beta$ and $\gamma$ position respectively, are predicted to be most stable in all SS form with $\varphi, \psi$ values in the right handed helical region and isopropylglycolide can also be realized in $\mathrm{R}, \mathrm{S}$ form. In substituted glycolides with cyclic side chains (aryl or alkyl) directly attach to $\mathrm{C}_{\alpha}$, the $\varphi, \psi$ values lie in the left helical regions for the most stable forms. Diphenylglycolide may be realized in all the forms, possibly due to the symmetric environment provided by phenyl rings to $\mathrm{C}_{\alpha}$ of backbone where as the $\varphi, \psi$ values for dicyclohexylglycolide corresponding to the most stable RR form lie in the left helical region with cyclohexyl moiety adopting the chair form. It is interesting to note from the results in Table 2 that the $\chi$ values adopted by side chain in diphenylglycolide depends upon the chain length i.e. it is trans in the dimer and gauche ${ }^{+}$in tetramer and hexamer where as the $\chi$ values in dicyclohexylglycolide are found to be independent of chain length in gauche ${ }^{+}$form. In both these substituted glycolides the cyclic (aromatic or aliphatic) side chain not only place restriction on the backbone conformation but are locked into the unfavorable gauche ${ }^{+}$.

The $\varphi, \psi$ values given in Table 1 and their combinations have been used for the generation various conformational states of polyglycolides of the form Ac$\left[\left\{\mathrm{O}-\mathrm{C}^{*} \mathrm{H}(\mathrm{X})-\mathrm{CO}\right\}_{2}\right]_{n}-\mathrm{OCH}_{3}$ with $n=1-3$ and their energies is computed by optimizing the torsion values up to $2^{\circ}$. Similar results are obtained with $n=2$ or 3 and hence only the results for $n=3$ are given in Table 3 . It is obvious from the results that substituted polyglycolide with smallest side chain i.e. polymethylglycolide is found to be most stable in the alternate $\mathrm{R}, \mathrm{S}$ or alternate $\mathrm{S}, \mathrm{R}$ forms with $\varphi, \psi$ values in the left $\&$ right or right \& left helical region. This observation is consistent with computational result by Witzke where the mesomeric form is found to be more stable than PLLA and PDLA [65]. The conformational behavior of polyphenylmethylglycolide because of the alternate lactic residue with small side chain is also found to be similar. The degeneracy of the alternate $\mathrm{R}, \mathrm{S}$ and alternate $\mathrm{S}, \mathrm{R}$ form implies their equal population. This very well explains the recent experimental observation that PLA homopolymer exhibit no CD signal in the wavelength range from 190 to $250 \mathrm{~nm}$ [66]. A molecular view of polymethylglycolide and polyphenylmethylglycolide in $\mathrm{R}, \mathrm{S}$ form shown in Figure $\mathbf{2}$ is similar in appearance to the 27 ribbon structure reported in poly $\Delta$ Leu peptides with $\Delta$ Leu residues being in alternate $\mathrm{Z}, \mathrm{E}$ or $\mathrm{E}, \mathrm{Z}$ forms 
Table 1. Conformational results ( $\varphi, \psi$, in degrees) for repeating units of polyglycolides.

\begin{tabular}{|c|c|c|c|c|c|c|}
\hline Form & 1 & 2 & $\Delta \mathrm{E}(\mathrm{kcal} / \mathrm{mol})$ & 1 & 2 & $\Delta \mathbf{E}(\mathrm{kcal} / \mathrm{mol})$ \\
\hline & \multicolumn{3}{|c|}{ Ac-dimethylglycolide $-\mathrm{OCH}_{3}$} & \multicolumn{3}{|c|}{ Ac-phenylmethylglycolide- $\mathrm{OCH}_{3}$} \\
\hline \multirow{5}{*}{ RS } & 60,30 & $-60,-30$ & 0.0 & 60,30 & $-60,-30$ & 0.0 \\
\hline & 30,30 & $-60,-30$ & 1.6 & 30,60 & $-30,120$ & 1.5 \\
\hline & 30,60 & $-30,-60$ & 1.9 & $30,-120$ & $-30,-60$ & 1.6 \\
\hline & 60,30 & $-90,0$ & 2.4 & 90,0 & $-30,-60$ & 1.7 \\
\hline & 30,60 & $-30,120$ & 3.0 & & & \\
\hline \multirow{4}{*}{ SR } & $-60,-30$ & 60,30 & 0.1 & $-60,-30$ & 60,30 & 0.9 \\
\hline & $-30,-60$ & 30,60 & 2.0 & $-90,0$ & 60,30 & 2.7 \\
\hline & $-30,-60$ & $30,-120$ & 2.4 & $-90,0$ & 90,0 & 3.7 \\
\hline & $-90,-30$ & 90,0 & 3.6 & $-90,0$ & 60,30 & 4.0 \\
\hline \multirow{5}{*}{ SS } & $-60,-30$ & $-60,0$ & 2.1 & $-60,-30$ & $-60,-30$ & 1.3 \\
\hline & $-60,-30$ & $-60,-30$ & 4.1 & $-90,0$ & $-90,0$ & 3.2 \\
\hline & $-90,0$ & $-90,0$ & 4.4 & $-30,-60$ & $-30,-60$ & 4.1 \\
\hline & $-60,-30$ & $-90,0$ & 4.5 & $-60,-30$ & $-90,0$ & 4.4 \\
\hline & $-90,0$ & $-60,-30$ & 4.7 & & & \\
\hline \multirow{5}{*}{$\mathbf{R R}$} & 60,30 & 60,30 & 2.3 & 60,30 & 60,30 & 2.1 \\
\hline & $-30,120$ & $30,-120$ & 4.2 & 90,0 & 90,0 & 4.0 \\
\hline & $30,-120$ & $30,-120$ & 4.3 & 30,60 & 30,60 & 4.9 \\
\hline & 30,60 & 30,60 & 4.4 & 90,0 & 60,30 & 5.2 \\
\hline & $-30,-60$ & 0,90 & 5.4 & & & \\
\hline & \multicolumn{3}{|c|}{ Ac-diisopropylglycolide- $\mathrm{OCH}_{3}$} & \multicolumn{3}{|c|}{ Ac-diisobutylylglycolide- $\mathrm{OCH}_{3}$} \\
\hline \multirow{3}{*}{ SS } & $-30,-60$ & $-30,-60$ & 0.0 & $-30,-60$ & $-60,-30$ & 0.0 \\
\hline & $-60,-30$ & $-30,-60$ & 1.8 & $-30,-60$ & $-30,-60$ & 1.1 \\
\hline & $-60,0$ & $-60,0$ & 2.1 & $-60,-30$ & $-60,-30$ & 2.0 \\
\hline \multirow{3}{*}{ RS } & 60,0 & $-30,-60$ & 0.5 & 60,0 & $-30,-60$ & 3.5 \\
\hline & 90,0 & $-30,-60$ & 0.9 & 60,30 & $-30,-60$ & 5.0 \\
\hline & 90,0 & $-60,-30$ & 2.6 & 30,60 & $-60,-30$ & 5.5 \\
\hline \multirow{3}{*}{ SR } & $-60,0$ & 60,30 & 2.1 & $-60,-30$ & 60,0 & 1.2 \\
\hline & $-90,0$ & 60,30 & 3.8 & $-30,-60$ & 60,0 & 3.9 \\
\hline & $-60,0$ & 60,0 & 3.9 & & & \\
\hline \multirow{4}{*}{ RR } & 60,0 & 60,0 & 2.9 & $30,-120$ & 150,60 & 6.5 \\
\hline & 60,0 & $30,-120$ & 4.4 & $30,-120$ & $30,-120$ & 8.9 \\
\hline & 60,180 & 60,0 & 5.4 & & & \\
\hline & $60,-150$ & $30,-120$ & 5.6 & & & \\
\hline
\end{tabular}




\begin{tabular}{|c|c|c|c|c|c|c|}
\hline & \multicolumn{3}{|c|}{ Ac-diphenylglycolide- $\mathrm{OCH}_{3}$} & \multicolumn{3}{|c|}{ Ac-dicyclohexylglycolide- $\mathrm{OCH}_{3}$} \\
\hline \multirow{6}{*}{$\mathbf{R R}$} & 30,60 & 60,30 & 0.0 & 60,30 & 60,30 & 0.0 \\
\hline & 30,60 & 30,60 & 0.7 & 90,0 & 60,30 & 2.5 \\
\hline & 30,60 & 0,90 & 1.9 & 90,0 & 90,0 & 4.9 \\
\hline & 60,30 & 0,90 & 2.3 & & & \\
\hline & 60,0 & 30,60 & 2.5 & & & \\
\hline & 90,0 & 30,60 & 2.6 & & & \\
\hline \multirow{5}{*}{ SS } & $-30,-60$ & $-60,-30$ & 0.1 & $-60,-30$ & $-60,-30$ & 43.1 \\
\hline & $-30,-60$ & $-30,-60$ & 0.7 & & & \\
\hline & $-30,-60$ & $-60,0$ & 1.2 & & & \\
\hline & $-60,-30$ & $-30,-60$ & 1.6 & & & \\
\hline & $-30,-60$ & $0,-90$ & 1.9 & & & \\
\hline \multirow{4}{*}{ SR } & $-60,0$ & 30,60 & 1.6 & $-60,-30$ & 90,60 & 24.0 \\
\hline & $-60,0$ & 60,30 & 2.5 & & & \\
\hline & $-90,0$ & 30,60 & 2.8 & & & \\
\hline & $-90,-30$ & 30,60 & 3.1 & & & \\
\hline \multirow{3}{*}{$\mathbf{R S}$} & 60,0 & $-30,-60$ & 1.6 & 30,60 & $-60,-30$ & 18.0 \\
\hline & 60,0 & $-60,-30$ & 2.5 & 30,60 & $-30,-60$ & 20.1 \\
\hline & 90,0 & $-30,-60$ & 3.1 & & & \\
\hline
\end{tabular}

and hence these structures are named as 27 ribbon type structures[67]. These structures are stabilized by the network of carbonyl-carbonyl interactions between the $\mathrm{i}^{\text {th }}$ residue and $\mathrm{i}^{\text {th }}+1$ residue $\left(\mathrm{d}_{\mathrm{O}_{\mathrm{i}}-\mathrm{C}_{\mathrm{i}+1}}=2.2+0.05 \AA\right.$ and $\left.\mathrm{d}_{\mathrm{O}_{i}-\mathrm{O}_{i+1}}=2.7 \pm 0.05 \AA\right)$. It may be mentioned that the splitting of carbonyl stretching in IR spectroscopy has also been attributed to the carbonyl-carbonyl interactions in polylactides [38]. In addition to the carbonyl- carbonyl interactions in polyphenylmethylglycolide, $\mathrm{C}-\mathrm{H} \cdots \pi$ interactions between methyl moiety of $\mathrm{i}^{\text {th }}$ residue and phenyl ring of both $\mathrm{i}^{\text {th }}-1$ and $\mathrm{i}^{\text {th }}+1$ residue are also observed. The importance of $\mathrm{C}-\mathrm{H} \cdots \pi$ interactions is well established in literature [68]. Graphical view in Figure 2 of polylactide in alternate R, S form clearly depicts that it adopts a structure with slight curvature where alternate methyl groups are placed on the either side of the backbone. Likewise, in polyphenylmethylglycolide, the phenyl moiety lies on one side and methyl group lies on the other side of the backbone. Such a disposition of side chains around the backbone may give rise to sheets like structure by association of two or more strands by hydrophobic interactions. A model building study involving two polylactide chain in the alternate $\mathrm{S}$, $\mathrm{R}$ form to give rise to a zipper like structure (Figure 3)
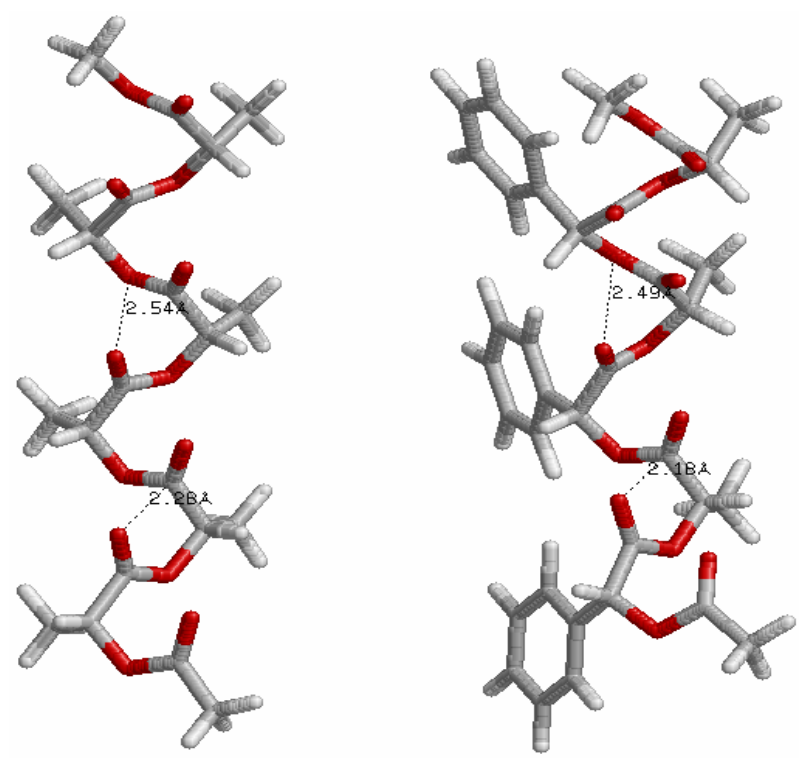

Figure 2. A molecular view of polylactide and polyphenylmethylglycolide in alternate $\mathrm{R}, \mathrm{S}$ form adopting 27 ribbon type structure stabilised by various interactions.

similar to leucine zipper in proteins in which carbonyl oxygen point on one side of the plane of molecule are 
Table 2. Most stable state $(\varphi, \psi, \omega, \chi$ in degrees) for substituted glycolides.

\begin{tabular}{|c|c|c|c|c|c|c|}
\hline Form & 1 & 2 & $\Delta \mathbf{E}(\mathbf{k c a l} / \mathbf{m o l})$ & 1 & 2 & $\Delta \mathbf{E}(\mathrm{kcal} / \mathrm{mol})$ \\
\hline & \multicolumn{3}{|c|}{ Ac-dimethylglycolide- $\mathrm{OCH}_{3}$} & \multicolumn{3}{|c|}{ Ac-phenylmethylglycolide- $\mathrm{OCH}_{3}$} \\
\hline $\mathbf{R S}$ & $\begin{array}{c}\mathbf{2 7 , 5 6} \\
174 \\
180\end{array}$ & $\begin{array}{c}-\mathbf{2 9}, \mathbf{- 5 8} \\
-174 \\
60\end{array}$ & 0.0 & $\begin{array}{c}\mathbf{2 6 , 5 6} \\
180 \\
175\end{array}$ & $\begin{array}{c}-\mathbf{3 5}, \mathbf{- 5 0} \\
-176 \\
60\end{array}$ & 0.0 \\
\hline SR & $\begin{array}{c}-\mathbf{2 9},-\mathbf{5 6} \\
-174 \\
60\end{array}$ & $\begin{array}{c}\mathbf{2 6 , 5 6} \\
174 \\
60\end{array}$ & 0.1 & $\begin{array}{c}\mathbf{- 5 2}, \mathbf{- 2 8} \\
-174 \\
-178\end{array}$ & $\begin{array}{c}\mathbf{2 5 , 5 8} \\
174 \\
180\end{array}$ & 1.1 \\
\hline SS & $\begin{array}{c}-\mathbf{6 0},-\mathbf{3 0} \\
-174 \\
60\end{array}$ & $\begin{array}{c}\mathbf{- 6 5}, \mathbf{- 1 0} \\
170 \\
180\end{array}$ & 2.3 & $\begin{array}{c}\mathbf{- 5 0 ,}, \mathbf{- 3 0} \\
176 \\
176\end{array}$ & $\begin{array}{c}\mathbf{- 2 4 ,}, \mathbf{- 5 6} \\
178 \\
60\end{array}$ & 1.6 \\
\hline $\mathbf{R R}$ & $\begin{array}{c}\mathbf{5 2 , 3 4} \\
172 \\
178\end{array}$ & $\begin{array}{l}\mathbf{2 2 , 5 6} \\
-174 \\
-178\end{array}$ & 2.6 & $\begin{array}{c}\mathbf{6 0 , 3 0} \\
180 \\
-179\end{array}$ & $\begin{array}{c}\mathbf{6 2 , 1 1} \\
164 \\
64\end{array}$ & 2.4 \\
\hline & \multicolumn{3}{|c|}{ Ac-diisopropylglycolide- $\mathrm{OCH}_{3}$} & \multicolumn{3}{|c|}{ Ac-diisobutylylglycolide- $\mathrm{OCH}_{3}$} \\
\hline SS & $\begin{array}{c}-\mathbf{2 5},-\mathbf{6 0} \\
180 \\
180(-60)\end{array}$ & $\begin{array}{c}-\mathbf{4 0},-\mathbf{7 0} \\
-172 \\
180(-60)\end{array}$ & 0.0 & $\begin{array}{c}-\mathbf{2 5},-\mathbf{6 0} \\
-172 \\
-40\end{array}$ & $\begin{array}{c}-\mathbf{2 0},-\mathbf{- 7 0} \\
-172 \\
-40\end{array}$ & 0.0 \\
\hline $\mathbf{R S}$ & $\begin{array}{c}\mathbf{6 0 , 0} \\
-176 \\
-65(55)\end{array}$ & $\begin{array}{c}\mathbf{- 2 5},-\mathbf{6 0} \\
180 \\
180(-60)\end{array}$ & 0.7 & $\begin{array}{c}\mathbf{4 5 , 2 5} \\
-178 \\
70\end{array}$ & $\begin{array}{c}\mathbf{- 2 5},-\mathbf{6 0} \\
-178 \\
-75\end{array}$ & 3.8 \\
\hline SR & $\begin{array}{c}-\mathbf{6 0}, \mathbf{0} \\
172 \\
-55(65)\end{array}$ & $\begin{array}{c}\mathbf{6 0 , 3 0} \\
176 \\
55(175)\end{array}$ & 2.5 & $\begin{array}{c}\mathbf{- 2 5},-\mathbf{6 0} \\
-172 \\
-75\end{array}$ & $\begin{array}{c}\mathbf{5 0 , 2 0} \\
-172 \\
75\end{array}$ & 1.5 \\
\hline $\mathbf{R R}$ & $\begin{array}{c}\mathbf{6 0}, \mathbf{1 0} \\
180 \\
-70(170)\end{array}$ & $\begin{array}{c}\mathbf{5 5 , 1 5} \\
180 \\
-70(170)\end{array}$ & 3.1 & $\begin{array}{c}\mathbf{5 0},-\mathbf{1 4 0} \\
178 \\
40\end{array}$ & $\begin{array}{c}\mathbf{1 6 0}, \mathbf{3 0} \\
-178 \\
175\end{array}$ & 6.8 \\
\hline & \multicolumn{3}{|c|}{ Ac-diphenylglycolide- $\mathrm{OCH}_{3}$} & \multicolumn{3}{|c|}{ Ac-dicyclohexylglycolide- $\mathrm{OCH}_{3}$} \\
\hline $\mathbf{R R}$ & $\begin{array}{c}\mathbf{3 5 , 5 0} \\
178 \\
173\end{array}$ & $\begin{array}{c}\mathbf{6 0 , 3 0} \\
-178 \\
170\end{array}$ & 0.0 & $\begin{array}{c}\mathbf{5 2 , 3 6} \\
178 \\
55\end{array}$ & $\begin{array}{c}\mathbf{5 2 , 3 6} \\
176 \\
55\end{array}$ & 0.0 \\
\hline SS & $\begin{array}{c}\mathbf{- 3 2},-\mathbf{5 4} \\
180 \\
178\end{array}$ & $\begin{array}{c}-\mathbf{6 0},-\mathbf{3 0} \\
180 \\
-173\end{array}$ & 0.3 & $\begin{array}{c}\mathbf{- 4 4},-\mathbf{3 2} \\
180 \\
94\end{array}$ & $\begin{array}{c}\mathbf{- 6 1}, \mathbf{- 2 5} \\
180 \\
85\end{array}$ & 43.1 \\
\hline SR & $\begin{array}{c}-\mathbf{3 8}, \mathbf{- 4 0} \\
178 \\
170\end{array}$ & $\begin{array}{c}\mathbf{3 0 , 5 6} \\
170 \\
172\end{array}$ & 0.3 & $\begin{array}{c}\mathbf{- 5 1 ,}-\mathbf{2 5} \\
176 \\
85\end{array}$ & $\begin{array}{c}84, \mathbf{6 3} \\
170 \\
62\end{array}$ & 24.6 \\
\hline $\mathbf{R S}$ & $\begin{array}{c}44,26 \\
-176 \\
180\end{array}$ & $\begin{array}{c}\mathbf{- 2 7},-\mathbf{5 8} \\
-176 \\
180\end{array}$ & 0.7 & $\begin{array}{c}\mathbf{2 9 , 5 6} \\
176 \\
59\end{array}$ & $\begin{array}{c}\mathbf{- 5 0},-\mathbf{2 7} \\
-172 \\
85\end{array}$ & 18.2 \\
\hline
\end{tabular}

$\varphi, \psi$ values are given in bold, $\omega$ in italics and $\chi_{1}, \chi_{2}$ in normal text.

accessible to water molecules for interaction. Possibly, polylactides in this type of sheet like structure are being used in encapsulation of drugs and mulch films.

The helical structures both left and right handed are found to be less stable in both polylactides and polyphenylmethylglycolides but the left handed helical structure is predicted to be more stable than right helical structure by at least $2 \mathrm{kcal}$ due to comparatively stronger hydrophobic interactions between the sides chains in the all RR form as compared to the all SS form. The $\mathrm{d}_{\mathrm{Oi}-\mathrm{Oi}+2}$ are found to be less than the sum of their van der Waal's radii in helices corresponding to RR/SS forms, suggesting the repulsion nature of this interaction and as apparent from $\mathrm{d}_{\mathrm{O}_{\mathrm{i}-\mathrm{O}_{i+2}}}(=2.20 \pm 0.02 \AA)$ values the repulsive interactions are negligible in the RS or SR forms. It is the magnitude of the attractive nature of carbonyl-carbonyl interactions and the repulsive forces between carbonyl oxygen of the $i^{\text {th }}$ residue and ester oxygen of $i^{\text {th }}+$ 2 and $i^{\text {th }}+3$ residues which dictate the structure adopted by polyglycolides. This also explains very well that the 
Table 3. Most stable conformers for substituted polyglycolides with $\varphi, \psi, \omega$ and $\chi$ being in degrees.

\begin{tabular}{|c|c|c|c|c|c|c|c|}
\hline \multicolumn{8}{|c|}{ Residue number } \\
\hline Form & 1 & 2 & 3 & 4 & 5 & 6 & $\Delta \mathbf{E}(\mathrm{kcal} / \mathrm{mol})$ \\
\hline \multicolumn{8}{|c|}{ Ac-(lactide) $)_{6}-\mathrm{OCH}_{3}$} \\
\hline RSRSRS & $\begin{array}{c}\mathbf{3 0 , 5 0} \\
178 \\
60\end{array}$ & $\begin{array}{c}\mathbf{- 3 5},-\mathbf{5 0} \\
-178 \\
60\end{array}$ & $\begin{array}{c}\mathbf{3 0 , 5 0} \\
178 \\
55\end{array}$ & $\begin{array}{c}\mathbf{- 3 5}, \mathbf{- 5 0} \\
-178 \\
65\end{array}$ & $\begin{array}{c}\mathbf{3 0 , 5 0} \\
178 \\
60\end{array}$ & $\begin{array}{c}\mathbf{- 3 5},-\mathbf{4 5} \\
180 \\
60\end{array}$ & 0.0 \\
\hline SRSRSR & $\begin{array}{c}-\mathbf{3 5},-\mathbf{5 0} \\
180 \\
60\end{array}$ & $\begin{array}{c}\mathbf{3 0 ,} \mathbf{5 0} \\
180 \\
60\end{array}$ & $\begin{array}{c}-\mathbf{3 5},-\mathbf{5 0} \\
-178 \\
60\end{array}$ & $\begin{array}{c}\mathbf{3 0 , 5 0} \\
178 \\
60\end{array}$ & $\begin{array}{c}-\mathbf{3 5},-\mathbf{5 0} \\
180 \\
55\end{array}$ & $\begin{array}{c}\mathbf{3 0 ,} \mathbf{5 0} \\
178 \\
55\end{array}$ & 0.4 \\
\hline RSSRRS & $\begin{array}{c}\mathbf{2 5 , 6 0} \\
170 \\
60\end{array}$ & $\begin{array}{c}-\mathbf{6 0},-\mathbf{3 0} \\
-178 \\
55\end{array}$ & $\begin{array}{c}\mathbf{- 3 5}, \mathbf{- 4 0} \\
172 \\
55\end{array}$ & $\begin{array}{c}\mathbf{3 0 , 5 5} \\
176 \\
65\end{array}$ & $\begin{array}{c}\mathbf{2 5 , 6 0} \\
172 \\
60\end{array}$ & $\begin{array}{c}\mathbf{- 3 5},-\mathbf{5 0} \\
-176 \\
60\end{array}$ & 2.0 \\
\hline RRRRRR & $\begin{array}{c}\mathbf{3 0}, \mathbf{5 0} \\
178 \\
60\end{array}$ & $\begin{array}{c}\mathbf{3 0 , 5 0} \\
178 \\
60\end{array}$ & $\begin{array}{c}\mathbf{3 0 , 5 0} \\
178 \\
60\end{array}$ & $\begin{array}{c}\mathbf{3 0 , 5 0} \\
178 \\
60\end{array}$ & $\begin{array}{c}\mathbf{3 0 , 5 0} \\
178 \\
60\end{array}$ & $\begin{array}{c}\mathbf{2 5 , 5 0} \\
178 \\
60\end{array}$ & 5.1 \\
\hline SSSSSS & $\begin{array}{c}\mathbf{- 3 5}, \mathbf{- 5 0} \\
178 \\
60\end{array}$ & $\begin{array}{c}-\mathbf{6 0},-\mathbf{3 0} \\
180 \\
60\end{array}$ & $\begin{array}{c}-\mathbf{6 0},-\mathbf{3 0} \\
180 \\
60\end{array}$ & $\begin{array}{c}\mathbf{- 3 5}, \mathbf{- 5 0} \\
180 \\
60\end{array}$ & $\begin{array}{c}\mathbf{- 3 5}, \mathbf{- 5 0} \\
180 \\
60\end{array}$ & $\begin{array}{c}\mathbf{- 3 5},-\mathbf{5 0} \\
180 \\
50\end{array}$ & 7.9 \\
\hline \multicolumn{8}{|c|}{ Ac-(phenylmethylglycolide) $)_{6}-\mathrm{OCH}_{3}$} \\
\hline RSRSRS & $\begin{array}{c}\mathbf{2 7}, \mathbf{5 6} \\
174 \\
30\end{array}$ & $\begin{array}{c}\mathbf{- 2 9},-\mathbf{5 8} \\
-170 \\
55\end{array}$ & $\begin{array}{c}\mathbf{2 2 , 6 4} \\
174 \\
30\end{array}$ & $\begin{array}{c}\mathbf{- 3 1}, \mathbf{- 6 0} \\
-168 \\
66\end{array}$ & $\begin{array}{c}\mathbf{4 1 ,} \mathbf{4 3} \\
172 \\
38\end{array}$ & $\begin{array}{c}\mathbf{- 3 6 , - 4 9} \\
-172 \\
58\end{array}$ & 0.0 \\
\hline SRSRSR & $\begin{array}{c}-\mathbf{3 1}, \mathbf{- 5 1} \\
180 \\
22\end{array}$ & $\begin{array}{c}\mathbf{2 8 , 5 6} \\
172 \\
60\end{array}$ & $\begin{array}{c}\mathbf{- 3 5}, \mathbf{- 4 5} \\
180 \\
30\end{array}$ & $\begin{array}{c}\mathbf{3 0 , 5 5} \\
172 \\
60\end{array}$ & $\begin{array}{c}-\mathbf{3 1},-\mathbf{5 1} \\
180 \\
20\end{array}$ & $\begin{array}{c}\mathbf{2 9 , 6 1} \\
174 \\
66\end{array}$ & 0.6 \\
\hline RSSRRS & $\begin{array}{c}\mathbf{3 0 , 5 5} \\
172 \\
55\end{array}$ & $\begin{array}{c}-\mathbf{6 0},-\mathbf{3 0} \\
-172 \\
60\end{array}$ & $\begin{array}{c}\mathbf{- 5 5},-\mathbf{2 5} \\
170 \\
60\end{array}$ & $\begin{array}{c}\mathbf{3 0 , 6 0} \\
-172 \\
70\end{array}$ & $\begin{array}{c}\mathbf{3 0 , 4 5} \\
176 \\
40\end{array}$ & $\begin{array}{c}-\mathbf{3 5},-\mathbf{5 0} \\
-172 \\
50\end{array}$ & 3.9 \\
\hline RRRRRR & $\begin{array}{c}\mathbf{6 0}, \mathbf{3 0} \\
162 \\
55\end{array}$ & $\begin{array}{c}\mathbf{5 2 , 2 0} \\
-172 \\
50\end{array}$ & $\begin{array}{c}\mathbf{5 0 , 3 0} \\
178 \\
68\end{array}$ & $\begin{array}{c}\mathbf{5 8 , 2 6} \\
-178 \\
54\end{array}$ & $\begin{array}{c}\mathbf{6 0 ,}, \mathbf{3 0} \\
172 \\
62\end{array}$ & $\begin{array}{c}\mathbf{5 8 , 2 0} \\
-172 \\
60\end{array}$ & 7.7 \\
\hline SSSSSS & $\begin{array}{c}\mathbf{- 6 0},-\mathbf{3 0} \\
180 \\
30\end{array}$ & $\begin{array}{c}\mathbf{- 6 0},-\mathbf{- 2 0} \\
180 \\
60\end{array}$ & $\begin{array}{c}\mathbf{- 4 0 ,}-\mathbf{3 5} \\
-174 \\
60\end{array}$ & $\begin{array}{c}\mathbf{- 7 5},-\mathbf{1 5} \\
-174 \\
60\end{array}$ & $\begin{array}{c}-\mathbf{6 0},-\mathbf{3 0} \\
-174 \\
60\end{array}$ & $\begin{array}{c}\mathbf{- 6 5},-\mathbf{- 1 0} \\
-172 \\
50\end{array}$ & 9.8 \\
\hline \multicolumn{8}{|c|}{ Ac-(isopropylglycolide) $6-\mathrm{OCH}_{3}$} \\
\hline SSSSSS & $\begin{array}{c}\mathbf{- 3 0},-\mathbf{5 5} \\
-172 \\
180(-60)\end{array}$ & $\begin{array}{c}\mathbf{- 3 0},-\mathbf{5 5} \\
-172 \\
180(-60)\end{array}$ & $\begin{array}{c}-\mathbf{3 0},-\mathbf{6 0} \\
-172 \\
180(-60)\end{array}$ & $\begin{array}{c}\mathbf{- 2 5},-\mathbf{6 0} \\
-172 \\
180(-60)\end{array}$ & $\begin{array}{c}-\mathbf{2 5},-\mathbf{6 0} \\
-176 \\
180(-60)\end{array}$ & $\begin{array}{c}-\mathbf{2 0},-\mathbf{6 5} \\
-172 \\
175(-65)\end{array}$ & 0.0 \\
\hline SRSRSR & $\begin{array}{c}-\mathbf{3 0}, \mathbf{- 5 5} \\
-172 \\
-80(40)\end{array}$ & $\begin{array}{c}\mathbf{6 5}, \mathbf{1 0} \\
172 \\
50(-70)\end{array}$ & $\begin{array}{c}-\mathbf{2 5},-\mathbf{6 5} \\
-174 \\
-80(40)\end{array}$ & $\begin{array}{c}\mathbf{6 5}, \mathbf{1 5} \\
170 \\
170(-70)\end{array}$ & $\begin{array}{c}-\mathbf{2 5},-\mathbf{6 0} \\
-172 \\
180(-60)\end{array}$ & $\begin{array}{c}\mathbf{6 0}, \mathbf{1 0} \\
178 \\
170(-70)\end{array}$ & 3.7 \\
\hline RSSRRS & $\begin{array}{c}\mathbf{5 0 , 3 0} \\
170 \\
-50(70)\end{array}$ & $\begin{array}{c}-\mathbf{2 5},-\mathbf{6 5} \\
-176 \\
180(-60)\end{array}$ & $\begin{array}{c}-\mathbf{3 0},-\mathbf{6 0} \\
-172 \\
-85(35)\end{array}$ & $\begin{array}{c}\mathbf{6 5 , 0} \\
-178 \\
175(-65)\end{array}$ & $\begin{array}{c}\mathbf{5 5}, \mathbf{1 5} \\
180 \\
175(-65)\end{array}$ & $\begin{array}{c}-\mathbf{2 0},-\mathbf{6 5} \\
-176 \\
180(-60)\end{array}$ & 4.2 \\
\hline RSRSRS & $\begin{array}{c}\mathbf{5 5 , 1 0} \\
-174 \\
-70(50)\end{array}$ & $\begin{array}{c}-\mathbf{3 0},-\mathbf{6 0} \\
-172 \\
-85(35)\end{array}$ & $\begin{array}{c}\mathbf{6 0}, \mathbf{1 0} \\
174 \\
170(-70)\end{array}$ & $\begin{array}{c}-\mathbf{2 5},-\mathbf{6 5} \\
-172 \\
-85(35)\end{array}$ & $\begin{array}{c}\mathbf{6 0 , 2 0} \\
174 \\
170(-70)\end{array}$ & $\begin{array}{c}-\mathbf{2 5},-\mathbf{6 0} \\
-172 \\
180(-60)\end{array}$ & 5.1 \\
\hline RRRRRR & $\begin{array}{c}\mathbf{6 0 , 0} \\
-172 \\
-60(60)\end{array}$ & $\begin{array}{c}\mathbf{6 0 , 0} \\
178 \\
-60(60)\end{array}$ & $\begin{array}{c}\mathbf{6 0 , 0} \\
178 \\
-60(60)\end{array}$ & $\begin{array}{c}\mathbf{6 5},-\mathbf{5} \\
178 \\
-60(60)\end{array}$ & $\begin{array}{c}\mathbf{6 0}, \mathbf{1 0} \\
180 \\
-60(60)\end{array}$ & $\begin{array}{c}\mathbf{6 0}, \mathbf{1 0} \\
180 \\
-60(60)\end{array}$ & 6.1 \\
\hline
\end{tabular}




\begin{tabular}{|c|c|c|c|c|c|c|c|}
\hline \multicolumn{8}{|c|}{ Ac-(isobutylglycolide) ${ }_{6}-\mathrm{OCH}_{3}$} \\
\hline SSSSSS & $\begin{array}{c}-\mathbf{3 0},-\mathbf{6 0} \\
-176 \\
-80\end{array}$ & $\begin{array}{c}-\mathbf{3 0},-\mathbf{6 0} \\
-174 \\
-75\end{array}$ & $\begin{array}{c}-\mathbf{2 5},-\mathbf{6 0} \\
-174 \\
-85\end{array}$ & $\begin{array}{c}-\mathbf{2 5},-\mathbf{6 0} \\
-172 \\
-85\end{array}$ & $\begin{array}{c}-\mathbf{2 5},-\mathbf{6 0} \\
-174 \\
-90\end{array}$ & $\begin{array}{c}-\mathbf{3 0},-\mathbf{6 0} \\
-172 \\
-90\end{array}$ & 0.0 \\
\hline SRSRSR & $\begin{array}{c}-\mathbf{3 0},-\mathbf{6 0} \\
-172 \\
-30\end{array}$ & $\begin{array}{c}\mathbf{3 5}, \mathbf{5 0} \\
172 \\
80\end{array}$ & $\begin{array}{c}-\mathbf{3 0},-\mathbf{5 5} \\
-172 \\
-150\end{array}$ & $\begin{array}{c}\mathbf{3 0 , 5 0} \\
172 \\
90\end{array}$ & $\begin{array}{c}-\mathbf{3 0},-\mathbf{6 0} \\
-172 \\
-30\end{array}$ & $\begin{array}{c}\mathbf{3 0 , 5 0} \\
174 \\
80\end{array}$ & 3.0 \\
\hline RRRRRR & $\begin{array}{c}\mathbf{2 5},-\mathbf{1 1 5} \\
180 \\
60\end{array}$ & $\begin{array}{c}\mathbf{1 5 0 ,} \mathbf{3 0} \\
180 \\
150\end{array}$ & $\begin{array}{c}\mathbf{2 5},-\mathbf{1 1 5} \\
180 \\
50\end{array}$ & $\begin{array}{c}\mathbf{1 5 0}, \mathbf{3 0} \\
-178 \\
170\end{array}$ & $\begin{array}{c}\mathbf{2 5}, \mathbf{- 1 1 5} \\
-178 \\
45\end{array}$ & $\begin{array}{c}\mathbf{1 5 5}, \mathbf{3 0} \\
-176 \\
170\end{array}$ & 9.0 \\
\hline RSRSRS & $\begin{array}{c}\mathbf{8 0},-\mathbf{3 0} \\
180 \\
80\end{array}$ & $\begin{array}{c}\mathbf{- 1 5},-\mathbf{- 7 5} \\
-172 \\
-80\end{array}$ & $\begin{array}{c}\mathbf{5 5 , 2 5} \\
170 \\
60\end{array}$ & $\begin{array}{c}\mathbf{- 1 5},-\mathbf{7 5} \\
180 \\
-115\end{array}$ & $\begin{array}{c}\mathbf{5 0 , 2 5} \\
174 \\
80\end{array}$ & $\begin{array}{c}\mathbf{- 2 0 ,}-\mathbf{6 0} \\
170 \\
-95\end{array}$ & 9.4 \\
\hline RSSRRS & $\begin{array}{c}\mathbf{3 0 ,} \mathbf{5 5} \\
180 \\
70\end{array}$ & $\begin{array}{c}\mathbf{- 2 5},-\mathbf{5 5} \\
170 \\
-100\end{array}$ & $\begin{array}{c}-\mathbf{3 0},-\mathbf{5 5} \\
-172 \\
-80\end{array}$ & $\begin{array}{c}\mathbf{3 5 , 5 0} \\
170 \\
125\end{array}$ & $\begin{array}{c}\mathbf{3 0 , 5 5} \\
170 \\
70\end{array}$ & $\begin{array}{c}-\mathbf{3 0}, \mathbf{- 4 5} \\
170 \\
-100\end{array}$ & 14.4 \\
\hline \multicolumn{8}{|c|}{ Ac-(phenylglycolide) ${ }_{6}-\mathrm{OCH}_{3}$} \\
\hline RRRRRR & $\begin{array}{c}\mathbf{4 5 ,}, \mathbf{3 5} \\
176 \\
38\end{array}$ & $\begin{array}{c}48,36 \\
-178 \\
47\end{array}$ & $\begin{array}{c}\mathbf{5 2 ,}, \mathbf{3 2} \\
-178 \\
47\end{array}$ & $\begin{array}{c}\mathbf{3 0 , 5 8} \\
-172 \\
55\end{array}$ & $\begin{array}{c}\mathbf{3 2 , 5 6} \\
176 \\
39\end{array}$ & $\begin{array}{c}\mathbf{2 8 , 5 6} \\
174 \\
41\end{array}$ & 0.0 \\
\hline RSSRRS & $\begin{array}{c}\mathbf{3 0 ,} \mathbf{5 0} \\
172 \\
45\end{array}$ & $\begin{array}{c}\mathbf{- 4 5}, \mathbf{- 3 5} \\
-172 \\
30\end{array}$ & $\begin{array}{c}\mathbf{- 5 5}, \mathbf{- 2 0} \\
-172 \\
55\end{array}$ & $\begin{array}{c}\mathbf{2 5 , 5 0} \\
-172 \\
60\end{array}$ & $\begin{array}{c}\mathbf{3 0 , 6 0} \\
-174 \\
55\end{array}$ & $\begin{array}{c}\mathbf{- 4 0 , - 4 5} \\
-172 \\
30\end{array}$ & 2.5 \\
\hline RSRSRS & $\begin{array}{c}\mathbf{2 4 , 6 0} \\
172 \\
50\end{array}$ & $\begin{array}{c}\mathbf{- 5 2},-\mathbf{3 0} \\
-172 \\
50\end{array}$ & $\begin{array}{c}\mathbf{2 4 , 6 0} \\
172 \\
56\end{array}$ & $\begin{array}{c}-\mathbf{5 2},-\mathbf{2 8} \\
-172 \\
50\end{array}$ & $\begin{array}{c}\mathbf{3 2 , 5 0} \\
174 \\
54\end{array}$ & $\begin{array}{c}\mathbf{- 5 4 , - 3 4} \\
-172 \\
56\end{array}$ & 8.8 \\
\hline SRSRSR & $\begin{array}{c}\mathbf{- 5 2},-\mathbf{2 8} \\
180 \\
50\end{array}$ & $\begin{array}{c}\mathbf{2 4 , 6 0} \\
172 \\
50\end{array}$ & $\begin{array}{c}\mathbf{- 5 2},-\mathbf{2 8} \\
-172 \\
50\end{array}$ & $\begin{array}{c}\mathbf{3 0 , 6 0} \\
172 \\
58\end{array}$ & $\begin{array}{c}-\mathbf{5 4}, \mathbf{- 2 8} \\
-172 \\
50\end{array}$ & $\begin{array}{c}\mathbf{3 4 , 5 0} \\
174 \\
50\end{array}$ & 11.6 \\
\hline SSSSSS & $\begin{array}{c}\mathbf{- 5 2},-\mathbf{2 8} \\
-172 \\
60\end{array}$ & $\begin{array}{c}\mathbf{- 5 2},-\mathbf{2 4} \\
-172 \\
60\end{array}$ & $\begin{array}{c}-\mathbf{6 8},-\mathbf{2 8} \\
-176 \\
60\end{array}$ & $\begin{array}{c}-\mathbf{5 4},-\mathbf{3 0} \\
-174 \\
60\end{array}$ & $\begin{array}{c}-\mathbf{6 6},-\mathbf{2 2} \\
-176 \\
60\end{array}$ & $\begin{array}{c}-\mathbf{6 0},-\mathbf{3 0} \\
-172 \\
60\end{array}$ & 12.7 \\
\hline \multicolumn{8}{|c|}{ Ac-(cyclohexylglycolide) $)_{6}-\mathrm{OCH}_{3}$} \\
\hline RRRRRR & $\begin{array}{c}\mathbf{3 5 , 5 0} \\
174 \\
61\end{array}$ & $\begin{array}{c}\mathbf{4 1 , 4 2} \\
174 \\
50\end{array}$ & $\begin{array}{c}\mathbf{4 8 , 4 3} \\
172 \\
65\end{array}$ & $\begin{array}{c}\mathbf{4 7 , 4 1} \\
172 \\
61\end{array}$ & $\begin{array}{c}\mathbf{5 0 ,}, \mathbf{3 5} \\
172 \\
60\end{array}$ & $\begin{array}{c}\mathbf{6 0 ,}, \mathbf{3 0} \\
-174 \\
51\end{array}$ & 0.0 \\
\hline RSRSRS & $\begin{array}{c}\mathbf{5 0 ,}, \mathbf{3 8} \\
178 \\
65\end{array}$ & $\begin{array}{c}-\mathbf{6 0},-\mathbf{3 0} \\
-166 \\
85\end{array}$ & $\begin{array}{c}\mathbf{3 0 , 6 0} \\
160 \\
65\end{array}$ & $\begin{array}{c}-\mathbf{6 0},-\mathbf{3 0} \\
-166 \\
85\end{array}$ & $\begin{array}{c}30, \mathbf{6 0} \\
162 \\
65\end{array}$ & $\begin{array}{c}-\mathbf{6 0},-\mathbf{3 0} \\
174 \\
85\end{array}$ & 153.0 \\
\hline SSSSSS & $\begin{array}{c}-\mathbf{6 0},-\mathbf{3 0} \\
-165 \\
85\end{array}$ & $\begin{array}{c}-\mathbf{6 0},-\mathbf{3 0} \\
180 \\
85\end{array}$ & $\begin{array}{c}-\mathbf{6 0},-\mathbf{3 0} \\
180 \\
85\end{array}$ & $\begin{array}{c}-\mathbf{6 0}, \mathbf{- 3 0} \\
180 \\
85\end{array}$ & $\begin{array}{c}-\mathbf{6 0},-\mathbf{3 0} \\
180 \\
85\end{array}$ & $\begin{array}{c}-\mathbf{6 0},-\mathbf{3 0} \\
180 \\
85\end{array}$ & 190.1 \\
\hline
\end{tabular}

$\varphi, \psi$ values are given in bold, $\omega$ in italics and $\chi_{1}, \chi_{2}$ in normal text.

enantiomeric form RSSRRS is more stable than the helical structures corresponding to all RR and all SS form but less stable than the alternate enantiomeric forms.

Polyisoproylglycolide and polyisobutylglycolide are found to be most stable in all SS form adopting the right handed helical structure without hydrogen bonds with $\varphi$, $\psi$ values of approximately $-30^{\circ},-60^{\circ}$ stabilized by various interactions shown in Figure 4. It is due to weaker hydrophobic interactions and the carbonyl-carbonyl in- teractions, the left handed helices in the all RR form is found to be less stable. Polyvaline and polyleucine peptides in the helical structure are used as surfactant by incorporating amino acid residue like lysine/aspartic or glutamic at termini positions. Similarly, polyisopropylglycolide and polyisobutylglycolide can also be exploited (by controlling chain length) as surfactant by incorporating charged amino acid residues at either terminus.

Polyphenylglycolide \& polycycloglycolide have been 


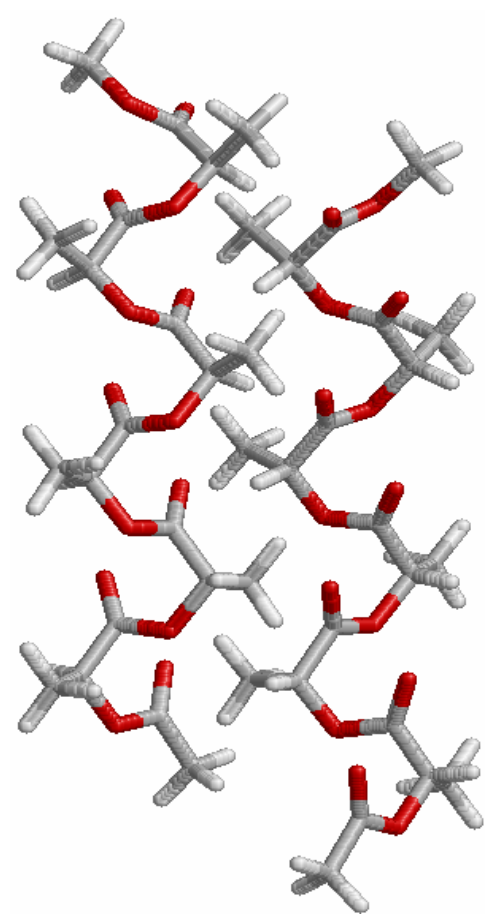

Figure 3. A model showing the formation of zipper like structure between two polylactides molecules in alternate $\mathrm{S}, \mathrm{R}$ form.

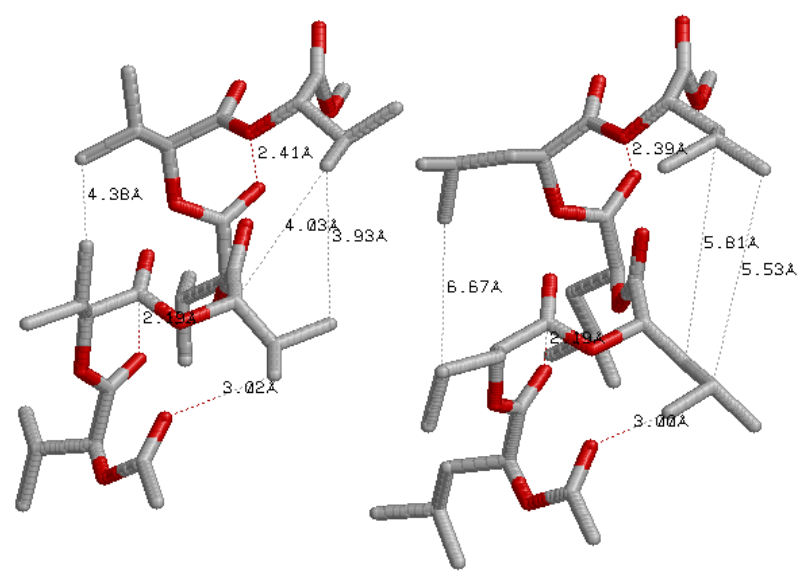

Figure 4. Polyisopropylglycolide and polyisobutylglycolide, without hydrogen bonds adopting right handed helical structure corresponding to most stable enatiomer in the SS form.

studied with emphasis on thermal \& hydrolytic degradation, rheological and visco elastic properties $[43,69]$. These polyglycolides are found to be most stable in the all RR form, adopting the left handed helical structures. In, Polyphenylglycolide the conformational state corresponding to the enantiomeric form RSSRRS lies within $2.5 \mathrm{kcal} / \mathrm{mol}$ of the most stable where as the conformations for pure enantiomeric forms lies higher in energy. The bulky side chains not only increase rotational barrier but also decrease the magnitude of carbonyl interactions.
On the basis of ${ }^{1} \mathrm{H}$ NMR results in methine region a high degree of stereo regularity for R, R-dicyclohexylglycolide has been reported and the isotactic nature of the polymer has been confirmed by ${ }^{13} \mathrm{C}$ NMR results [70]. The computational results together with graphical view shown in Figure 5 clearly depict the identical and equivalent environment for methine moieties in the helical structure. Thus, the conformational results are not only in agreement with the reported experimental finding but provide more information about the adopted structure \& the interactions which stabilise the structure. The computational results also reveal that these molecules cannot be realized in the all SS form because of stearic clash between the side chains of $i^{\text {th }}$ and $i^{\text {th }}+3$ residues.

\subsection{Modelling of Carbonyl-Carbonyl Interactions}

Based on a systematic study between ketonic groups in the Cambridge structure database carbonyl-carbonyl interactions [71,72] have been modeled by three main types of interaction motifs. Importance of carbonyl-carbonyl interactions as a stabilizing factor in $\alpha$-helices, $\beta$-sheets and right-handed twist is well-documented $[73,74]$ and are substantiated by interacting two acetone molecules in different geometries. In one of the geometries, the carbonyl groups of two acetone molecules are vertically above each other with their carbonyl groups aligned in an antiparallel direction, whereas in the other, one acetone molecule's carbonyl group approaches the carbonyl group of the second molecule in a perpendicular direction. As expected, the interaction energy corresponding to the first geometry was found to be approximately $\sim 45 \mathrm{kcal} \cdot \mathrm{mol}^{-1}$ and may not reflect the quantitative nature of the carbonyl-carbonyl interactions, as this model envisages a pair of such interactions without any repulsion. The interaction energy, $\Delta \mathrm{E}$ found to be between 4 and $10 \mathrm{kcal} \mathrm{mol}^{-1}$ depending upon the approach of one acetone molecule over the length of the carbonyl group of the second acetone molecule in the perpendicular direction. The carbonyl-carbonyl interactions also stabilize the partially allowed Ramachandran conformations of aspartic acid and asparagines [75] and helical structures without hydrogen bonds in peptides constructed from achiral and unusual amino acids $[67,76$, 77].

\subsection{Interactions}

The right handed helical structure corresponding to the all SS form in polyisopropylglycolide and polyisobutylglycolide shown in Figure $\mathbf{4}$ are stablised by carbonyl carbonyl interactions and $\mathrm{C}-\mathrm{H}-\mathrm{O}$ interactions 

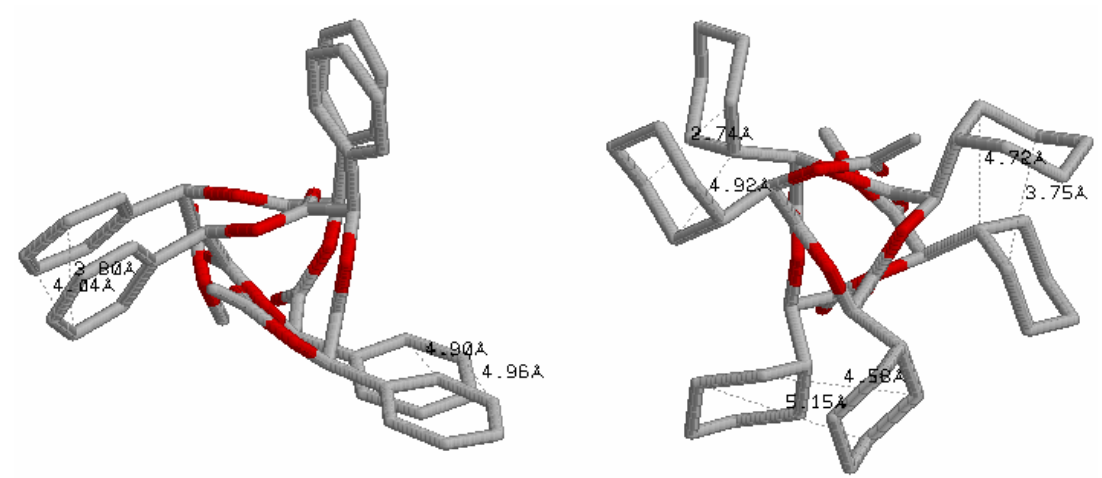

Figure 5. Formation of left handed helical structure in the most stable enatiomeric form (RR) in polymandelide and polydicyclohexylglycolide stabilized by various interactions.

between $\mathrm{C}_{\gamma} / \mathrm{C}_{\delta}$ of methyl group of $\mathrm{i}^{\text {th }}+3$ residue side chain with carbonyl oxygen of $i^{\text {th }}$ residue. In polyisobutylglycolide, $\mathrm{C}-\mathrm{H}-\mathrm{O}$ interactions are also noticed between $\mathrm{C}_{\beta}-\mathrm{H}$ of $\mathrm{i}^{\text {th }}+3$ residue and carbonyl oxygen of $\mathrm{i}^{\text {th }}$ residue (Figure 4). The $\mathrm{C}-\mathrm{H}-\mathrm{O}$ interactions are well documented and discussed in detail for inclusion complexes, as determinant of stability and specificity in trans membrane helix and in biological structure. $[79,80] \mathrm{Hy}-$ drophobic interactions are found to be operative in polyisopropylglycolide but absent in polyisobutylglycolide. The magnitude of carbonyl-carbonyl interactions appears to be similar in polylactides and polyisopropylglycolides on the basis of distance $\left(\mathrm{d}_{\mathrm{O}_{\mathrm{i}}-\mathrm{C}_{\mathrm{i}+1}}=2.4-2.6\right.$ $\AA$ ). The distance between carbonyl oxygen's of $i^{\text {th }}$ and ester oxygen of $\mathrm{i}^{\text {th }}+3$ residue lies in the range 2.3 to 2.5 $\AA$. The van der Waal radius of oxygen is $1.45 \AA$ and thus the repulsive interactions between carbonyl oxygen's of $i^{\text {th }}$ and ester oxygen of $i^{\text {th }}+3$ residues may be operative. The stability of any state arises due to the net result of attractive and repulsive interactions and thus, may influence the magnitude of glass transition temperature.

A molecular view of polyphenylglycolide and polycyclohexylglycolide in the left helical structure shown in Figure 5 reveals that both these molecules are stabilized by the network of carbonyl-carbonyl interactions $\left(\mathrm{d}_{\mathrm{O}_{\mathrm{i}-1} \cdots \mathrm{C}_{\mathrm{i}}}\right.$ $=2.2$ to $2.7 \AA$, but weaker as compare to corresponding form in polylactides) and by $\mathrm{C}-\mathrm{H}-\mathrm{O}$ interactions between the carbonyl oxygen of $\mathrm{i}^{\text {th }}-1$ residue and $\mathrm{C}_{\alpha}$ of $\mathrm{i}^{\text {th }}$ +2 residue (in polyphenylglycolide) and $\mathrm{C}_{\gamma}-\mathrm{H}$ and $\mathrm{C}_{\beta}-\mathrm{H}$ of $\mathrm{i}^{\text {th }}+2$ residue with carbonyl oxygen of $\mathrm{i}^{\text {th }}-1\left(\mathrm{~d}_{\mathrm{O}-\mathrm{H}}=\right.$ $2.4-2.6 \AA$ ) (in cyclohexylglycolide). In addition to these interactions, the $\mathrm{C}_{\beta-\gamma-\delta}$ face of aromatic ring of the $\mathrm{i}^{\text {th }}$ residue is involved in stacking interactions with the $\mathrm{C}_{\gamma-\delta-\xi}$ face of aromatic ring of the $\mathrm{i}^{\text {th }}+3$ residues in polyphenylglycolide and the stacking interactions are usually stronger than the hydrophobic interactions. The hydrophobic interactions between the side chain of $i^{\text {th }}$ and $i^{\text {th }}+$ 3 residues also contribute towards the stability of this state in polycyclohexylglycolide. The carbonyl- carbonyl interactions are operative in all the conformations of all forms of substituted polyglycolides with highly shielded parallel motif with only one short carbon-oxygen interacttion. The quiet high $T_{g}$ of polyphenylglycolide and polyc-yclohexylglycolide can be explained in terms of the network of the these interactions. Polylactides lack this type of network of interactions and that may be the reason for their is comparatively low $\mathrm{T}_{\mathrm{g}}$. Thus, the nature of non-covalent interactions by proper choice of side chains and chirality can be exploited for the construction and designing of polymers in required handed structures.

\subsection{Simulations}

The conformational results for various polyglycolides after 1 ns simulation in water are summarized in Table 4. The molecular view of polylactide (Figure 6(a)) with water molecules within $3 \AA$ of its surface clearly show that almost all of the carbonyl oxygen are involved in interactions with water molecules $\left\{\mathrm{d}_{\mathrm{O}-\mathrm{H}}=1.5-2.1\right.$ and $\mathrm{d}_{\mathrm{O}-\mathrm{O}}=2.4-3.0 \AA$ and the angle $\angle \mathrm{O} \cdots \mathrm{H}-\mathrm{O}$ being in the range $149.9^{\circ}-174.7^{\circ}$ \}. This observation is consistent with the fact that in water, ester bond undergo hydrolysis and thus provides explains for the use of polylactides as sutures, mulch films etc. The degradation products of polylactides are non-toxic as lactate is produced in body by the process of anaerobic glycolysis. Similar results were obtained for polyphenylmethylglycolide.

Polyisopropylglycolide and polyisobutylglycolide having branching at $\beta$ and $\gamma$ positions respectively exhibit almost similar behaviour on simulation in water. The results in Table 4 reveal that due to the interaction of water molecules with backbone, consecutive turns (analogous to that in proteins) of the type VIa, Type I and $\gamma$ are introduced at the $\mathrm{C}$ terminal in polyisopropulglycolide. As a function of simulation time the molecule start 
Table 4. Simulations results for substituted polyglycolides after $1 \mathrm{~ns}$ simulation in water.

\begin{tabular}{|c|c|c|c|c|c|c|c|c|c|c|}
\hline \multicolumn{11}{|c|}{ Residue no } \\
\hline poly glycolide & 1 & 2 & 3 & 4 & 5 & 6 & 7 & 8 & 9 & 10 \\
\hline methyl & $\begin{array}{c}\mathbf{6 4},-\mathbf{8 5} \\
-164\end{array}$ & $\begin{array}{c}-\mathbf{6 5}, \mathbf{1 2 3} \\
-177\end{array}$ & $\begin{array}{c}\mathbf{5 7 , 3 4} \\
-173\end{array}$ & $\begin{array}{c}-\mathbf{5 7}, \mathbf{1 0 8} \\
-178\end{array}$ & $\begin{array}{c}\mathbf{1 0 0},-\mathbf{3 5} \\
178\end{array}$ & $\begin{array}{c}-\mathbf{6 4}, \mathbf{1 1 4} \\
-151\end{array}$ & $\begin{array}{c}\mathbf{8 7},-\mathbf{3 9} \\
174\end{array}$ & $\begin{array}{c}-74,-27 \\
-169\end{array}$ & $\begin{array}{c}\mathbf{6 7},-\mathbf{1 2 4} \\
178\end{array}$ & $\begin{array}{c}\mathbf{- 5 9 , - 2 6} \\
-166\end{array}$ \\
\hline phenyl/methyl & $\begin{array}{c}\mathbf{6 3},-\mathbf{- 1 2 3} \\
180\end{array}$ & $\begin{array}{c}-\mathbf{7 3},-\mathbf{5} \\
-179\end{array}$ & $\begin{array}{c}\mathbf{1 3 6}, \mathbf{3 6} \\
-164\end{array}$ & $\begin{array}{c}-\mathbf{7 9},-10 \\
-177\end{array}$ & $\begin{array}{c}\mathbf{7 3},-\mathbf{1 5 4} \\
167\end{array}$ & $\begin{array}{c}\mathbf{- 6 3},-\mathbf{1 5} \\
154\end{array}$ & $\begin{array}{c}\mathbf{5 3},-\mathbf{1 6 2} \\
-174\end{array}$ & $\begin{array}{c}-\mathbf{7 2},-\mathbf{2 2} \\
-179\end{array}$ & $\begin{array}{c}\mathbf{1 7 6}, \mathbf{4 4} \\
-178\end{array}$ & $\begin{array}{c}-\mathbf{1 0 3}, \mathbf{2 5} \\
168\end{array}$ \\
\hline isopropyl & $\begin{array}{c}-\mathbf{7 5},-\mathbf{4} \\
-179 \\
-83(48)\end{array}$ & $\begin{array}{c}-84,14 \\
171 \\
-76(49)\end{array}$ & $\begin{array}{c}\mathbf{- 1 3 4}, \mathbf{- 2 8} \\
-171 \\
-31(-153)\end{array}$ & $\begin{array}{c}-\mathbf{9 0 ,}, 35 \\
173 \\
-86(30)\end{array}$ & $\begin{array}{c}-\mathbf{6 5}, \mathbf{1 1 4} \\
-168 \\
-87(31)\end{array}$ & $\begin{array}{c}-\mathbf{4 8},-\mathbf{9} \\
175 \\
-56(67)\end{array}$ & $\begin{array}{c}-\mathbf{8 8},-\mathbf{2 1} \\
170 \\
-72(54)\end{array}$ & $\begin{array}{c}-\mathbf{8 0},-\mathbf{3 7} \\
144 \\
-176(64)\end{array}$ & $\begin{array}{c}-\mathbf{9 6 , 8 0} \\
-171 \\
-51(-178)\end{array}$ & $\begin{array}{c}-84,156 \\
-179 \\
-72(50)\end{array}$ \\
\hline isobutyl & $\begin{array}{c}-\mathbf{6 8}, \mathbf{- 3 6} \\
175 \\
-63\end{array}$ & $\begin{array}{c}-77,-5 \\
172 \\
-58\end{array}$ & $\begin{array}{c}\mathbf{- 9 2 ,}, \mathbf{3 2} \\
176 \\
-65\end{array}$ & $\begin{array}{c}-\mathbf{6 9},-\mathbf{4} \\
166 \\
-70\end{array}$ & $\begin{array}{c}-\mathbf{9 7}, \mathbf{- 2 4} \\
174 \\
-147\end{array}$ & $\begin{array}{c}-\mathbf{6 7},-\mathbf{1 6} \\
164 \\
-56\end{array}$ & $\begin{array}{c}-\mathbf{8 0},-\mathbf{2 8} \\
-178 \\
-168\end{array}$ & $\begin{array}{c}-\mathbf{7 7},-\mathbf{- 1 4} \\
169 \\
-90\end{array}$ & $\begin{array}{c}-\mathbf{7 7},-\mathbf{2 1} \\
164 \\
-76\end{array}$ & $\begin{array}{c}\mathbf{- 7 3},-\mathbf{1 7} \\
179 \\
-86\end{array}$ \\
\hline phenyl & $\begin{array}{c}\mathbf{8 8 ,} \mathbf{6} \\
171 \\
99\end{array}$ & $\begin{array}{c}\mathbf{6 8 , 1 8} \\
150 \\
137\end{array}$ & $\begin{array}{c}\mathbf{6 8 , 2 6} \\
-177 \\
126\end{array}$ & $\begin{array}{c}\mathbf{7 5 , 1 2} \\
-178 \\
118\end{array}$ & $\begin{array}{c}\mathbf{1 0 2 , 1} \\
-172 \\
99\end{array}$ & $\begin{array}{c}\mathbf{1 2 8},-\mathbf{- 1 0} \\
-179 \\
97\end{array}$ & $\begin{array}{c}\mathbf{7 4 ,}, \mathbf{3 2} \\
170 \\
108\end{array}$ & $\begin{array}{c}\mathbf{5 7}, \mathbf{3 8} \\
-176 \\
147\end{array}$ & $\begin{array}{c}\mathbf{8 6 ,}, 32 \\
-171 \\
113\end{array}$ & $\begin{array}{c}\mathbf{1 4 9 , 6 8} \\
175 \\
138\end{array}$ \\
\hline cyclohexyl & $\begin{array}{c}\mathbf{5 3 ,}, \mathbf{3 5} \\
-168 \\
54\end{array}$ & $\begin{array}{c}\mathbf{7 0 , 1 8} \\
179 \\
73\end{array}$ & $\begin{array}{c}\mathbf{8 7}, \mathbf{1 8} \\
-158 \\
59\end{array}$ & $\begin{array}{c}\mathbf{1 0 4 , 2 2} \\
-175 \\
64\end{array}$ & $\begin{array}{c}77, \mathbf{3 6} \\
-176 \\
32\end{array}$ & $\begin{array}{c}\mathbf{6 0 , 2 3} \\
-165 \\
49\end{array}$ & $\begin{array}{c}\mathbf{9 2 ,}-\mathbf{2 1} \\
180 \\
49\end{array}$ & $\begin{array}{c}\mathbf{8 9 ,}, \mathbf{4 1} \\
-153 \\
74\end{array}$ & $\begin{array}{c}\mathbf{6 5 , 4 0} \\
174 \\
43\end{array}$ & $\begin{array}{c}\mathbf{9 0},-\mathbf{1 0 4} \\
169 \\
44\end{array}$ \\
\hline
\end{tabular}

$\varphi, \psi$ values are given in bold, $\omega$ in italics and $\chi_{1}, \chi_{2}$ in normal text.

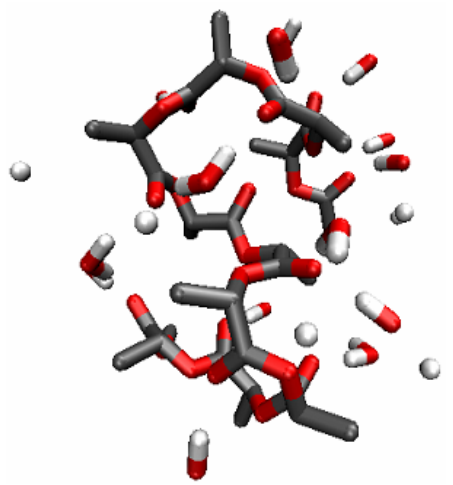

(a)

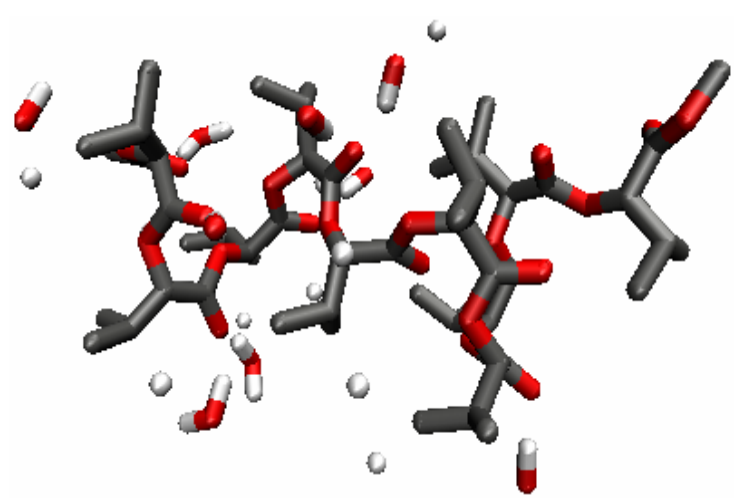

(b)

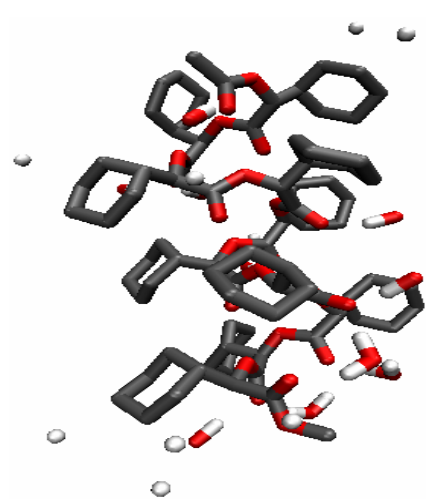

(c)

Figure 6. Molecular view of substituted glycolides after $1 \mathrm{~ns}$ simulation in water with the interacting water molecules within $3 \AA$ of the substituted glycolides. (a) Polylactide; (b) polyisopropylglycolide; (c) polycyclohexylglycolide.

adopting compact structure with decrease in the number of water molecules interacting with backbone with the increase in hydrophobic interactions between the side chains. The molecular view of polyisopropylglycolide after 1ns simulation in water is shown in Figure 6(b) reveals that the branching at $\beta$ position protects the backbone from approach of water molecules.

The backbone $\varphi, \psi$ angles, both for polyphenylglycolide and polycyclohexylglycolide on simulation also remain in the left handed helical region. The left helical structure in polycyclohexylglycolide shown in Figure 6(c) is characterized by average $\varphi, \psi$ values of 49.5 , $27.5^{\circ}$ analogous to the $3_{10}$ helix in peptides, rotation per residue $\theta=100^{\circ}$, rise per residue $h=-1.68 \AA$. It is worth mentioning here that by circular dichroism measurements it has been shown, poly (D-dicyclohexyl glycine) forms more stable and ordered structure due to stronger non covalent interactions in water, ethanol or triluoroethanol in comparison with poly (D-phenylglycine) [81]. In polyphenylglycolide, due to interaction of water molecules with backbone, a kink is introduced in the middle. The analysis of simulation results for polyisobutylglycolide, polyphenylglycolide and polycyclohexylglycolide reveals that numbers of water molecules interacting with backbone carbonyl group are found to remain constant as a function of simulation time having preference for the $\mathrm{C}$ terminal. This may implies that the hydrolysis of these polyglycolides may start from $\mathrm{C}$ ter- 
minal. Both, in case of polyphenylglycolide and polycyclohexylglycolide, the number of water molecules protruding to interact with backbone is less in comparison to other substituted polyglycolides. This may be regarded as that these glycolides are more resistant to water hydrolysis.

\section{CONCLUSIONS}

The conformational behavior of substituted polyglycolides has been found to depend on the nature of side chains. Polylactide and polyphenylmethylglycolide can be realized in both the enantiomeric forms $\mathrm{R}, \mathrm{S} / \mathrm{S}, \mathrm{R}$ forms with $\varphi, \psi$ values giving rise to the $2_{7}$ ribbon like structure which are stabilised by carbonyl-carbonyl interactions. Polyisopropylglycolide and isobutylglycolide having branching at $\beta \& \gamma$ respectively in their side chains it is the all $\mathrm{S}$ form which is observed to be the most stable with $\varphi, \psi$ values lying in right handed helical region of Ramachandran plot stablised by hydrophobic interactions. Both polyphenylglycolide and polycyclohexylglycolide, having cyclic side chain directly attach to $\mathrm{C}_{\alpha}$ group of backbone are found to be most stable in the all $\mathrm{R}$ form and adopt left handed helical structure stabilised by stacking interactions and hydrophobic interactions respectively. It is nature and magnitude of various interactions dictate the most stable form of polyglycolides and their structure together with handedness. Simulation studies in water reveal that the approach of water molecules to the backbone depends on the bulkiness of side chain and the adopted structure.

\section{REFERENCES}

[1] Tsuji, H. and Sumida, K.J. (2001) Poly(L-lactide). V. Effects of storage in swelling solvents on physical properties and structure of poly(L-lactide). Journal of Applied Polymer Science, 79, 1582.

doi:10.1002/1097-4628(20010228)79:9<1582::AID-APP 60>3.0.CO;2-7

[2] Bleach, N.C., Tanner, K.E., Kellomaki, M. and Tormala, P. (2001) Effect of filler type on the mechanical properties of self reinforced polylactide calcium phosphate composites. Journal of Materials Science: Materials in Medicine, 12, 911-915. doi:10.1023/A:1012884310027

[3] Ikada, Y. and Tsuji, H. (2000) Biodegradable polyesters for medical and ecological applications. Macromolecular Rapid Communications, 21, 117. doi:10.1002/(SICI)1521-3927(20000201)21:3<117::AID -MARC117>3.0.CO;2-X

[4] Dorgan, J.R., Lehermeier, H.J., Palade, L.-I. and Ciero, J. (2001) Polylactides: Properties and prospects of an environmentally benign plastic form renewable resources. Macromolecular Symposia, 175, 55. doi:10.1002/1521-3900(200110)175:1<55::AID-MASY5 5>3.0.CO;2-K

[5] Lehermeir, H.J., Dorgan, J.R. and Way, D.J. (2001) Gas permeation properties of poly(lactic acid). Journal of Membrane Science, 190, 243.

doi:10.1016/S0376-7388(01)00446-X

[6] Benicewicz, B.C. and Hopper, P.K. (1990) Polymers for absorbable surgical sutures-Part I. Journal of Bioactive and Compatible Polymers, 5, 453-472. doi: $10.1177 / 088391159000500407$

[7] Aluminum Association (1999) Tokoyo steps up to recycling efforts.

www.mmc.co.jp/english/enviroment/environment1999.p df.

[8] Vert, M. (2000) Lactide polymerization faced with therapeutic application requirements. Macromolecular Symposia, 153, 333-342.

doi:10.1002/1521-3900(200003)153:1<333::AID-MASY 333>3.0.CO;2-9

[9] Zhang, L., Xiong, C. and Deng, X. (1995) Biodegradable polyester blends for biomedical application. Journal of Applied Polymer Science, 56, 103. doi:10.1002/app.1995.070560114

[10] Mochizuki, M. (2002) Properties and application of aliphatic polyester products. In: Doi, Y. and Steinbüchel, A., Eds., Biopolymers Polyesters III. Applications and Commercial Products, 1st Edition, Wiley-VCH, Weinheim, $1-23$.

[11] Tsuji, H. (2002) Polylactides. In: Doi, Y. and Steinbüchel, A., Eds., Biopolymers Polyesters III. Applications and Commercial Products, 1st Edition, Wiley-VCH, Weinheim, 129-177.

[12] Ikada, Y. (1999). Polymeric biomaterials research. Advanced Engineering Materials, 1, 67-68. doi:10.1002/(SICI) 1527-2648(199909)1:1<67::AID-AD EM67>3.0.CO;2-E

[13] Duek, E.A.R., Zavaglia, C.A.C. and Belangero, W.D. (1999) In vitro study of poly(lactic acid) pin degradation. Polymer, 40, 6465. doi:10.1016/S0032-3861(98)00846-5

[14] Coombes, A.G.A. and Meikle, M.C. (1994) Resorbable polymers as replacements for bone grafts. Clinical Materials, 17, 35-67. doi:10.1016/0267-6605(94)90046-9

[15] Cha, Y. and Pitt, C.G. (1990) The biodegradability of polyester blends. Biomaterials, 11, 108-112. doi:10.1016/0142-9612(90)90124-9

[16] Ouchi, T. and Ohya, Y. (2004) Design of lactide copolymers as biomaterials. Journal of Polymer Science Part A: Polymer Chemistry, 42, 453-462. doi: $10.1002 /$ pola. 10848

[17] Hoogsteen, W., Postema, A.R. and Pennings, A.J. (1990) Crystal structure, conformation, and morphology of solution-spun poly(L-lactide) fibers. Macromolecules, 23, 634-642. doi:10.1021/ma00204a041

[18] Vert, M., Schwarch, G. and Coudane, J. (1995) Present and future of PLA polymers. Journal of Macromolecular Science: Pure and Applied Chemistry, 32, 787-796. doi:10.1080/10601329508010289

[19] Mainil-Varlet, P., Rahm, R. and Gogolewski, S. (1997) Long-term in vivo degradation and bone reaction to various polylactides. Biomaterials, 18, 257-266. doi:10.1016/S0142-9612(96)00126-3

[20] Vert, M., Li, S., Spenlehauer, G. and Guerin, P. (1992) Bioresorbability and biocompatibility of aliphatic polyesters. Journal of Materials Science: Materials in Medicine, 3, 432-446. doi: 10.1007/BF00701240 
[21] Duncan, R. and Kopecek, J. (1984) Soluble synthetic polymers as potential drug carriers. Advances in Polymer Science, 57, 51-101.

[22] Gruber, P.R. and Brien, M.O. (2002) Polylactides nature works TMPLA. In: Doi, Y. and Steinbüchel, A., Eds., Biopolymers Polyesters III. Applications and Commercial Products, 1st Edition, Wiley-VCH, Weinheim, 235250.

[23] Kawahima, N., Ogawa, S., Obuchi, S., Matsuo, M. and Yagi, T. (2002) Polylactic acid "LACEA", In: Doi, Y. and Steinbüchel, A. Eds., Biopolymers Polyesters III. Applications and Commercial Products, 1st Edition, WileyVCH, Weinheim, 251-274.

[24] Eling, B., Gogolewski, S. and Pennings, A.J. (1982) Comparison of melt-spun and solution-spun 1-form fibers. Polymer, 23, 1587. doi:10.1016/0032-3861(82)90176-8

[25] Tsuji, H., Smith, R., Bonfield, W. and Ikada, Y. (2000) Porous biodegradable polyesters. I. Preparation of porous poly(L-lactide) films by extraction of poly(ethylene oxide) from their blends. Journal of Applied Polymer Science, 75, 629-639.

doi:10.1002/(SICI)1097-4628(20000131)75:5<629::AID -APP5>3.0.CO;2-A

[26] Fambri, L., Pegoretti, A., Fenner, R., Incardona, S.D. and Migliaresi, C. (1997) Processing and in vitro degradation of poly(L-lactic acid) fibres. Polymer, 38, 79 . doi:10.1016/S0032-3861(96)00486-7

[27] Perepelkin, K.E. (2002) Chemistry and technology of chemical fibers. Poly(lactide) fibers: Fabrication, properties, use, prospects, a review. Fibre Chemistry, 34, 85. doi:10.1023/A:1016359925976

[28] Conn, R.E., Kolstad, J.J., Borzelleca, J.F., Dixler, D.S., Filer, L.J., LaDu, B.N. and Pariza, M.W. (1995) Safety assessment of polylactide (PLA) for use as a food-contact polymer. Food and Chemical Toxicology, 33, 273-283. doi:10.1016/0278-6915(94)00145-E

[29] Li, T., Strung, S., Radke, W., Klein, R. and Hofe, T. (2011) Chromatographic separation of polylactides by stereochemical composition. Polymer, 52, 40-45. doi:10.1016/j.polymer.2010.10.056

[30] Drumlight, R.E., Gruber, P.R. and Henton, D.E. (2000) Polylactic acid technology. Advanced Materials, 12, 1841-1846.

doi:10.1002/1521-4095(200012)12:23<1841::AID-ADM A1841>3.0.CO;2-E

[31] Dattaa, R., Tsaia, S.-P., Bonsignorea, P., Moona, S.-H. and Frank, J.R. (1995) Technological and economic potential of polylactic acid and lactic acid derivatives. FEMS Microbiology Reviews, 16, 221-231. doi:10.1111/j.1574-6976.1995.tb00168.x

[32] Yang, X., Kang, S., Yang, Y., Aou, K. and Hsu, S.L. (2004) Raman spectroscopic study of conformational changes in the amorphous phase of poly(lactic acid) during deformation. Polymer, 45, 4241-4248. doi:10.1016/i.polymer.2004.03.107

[33] Sarasua, J.R., Rodríguez, N.L., Arraiza, A.L. and Meaurio, E. (2005) Stereoselective crystallization and specific interactions in polylactides. Macromolecules, 38, 8362-8365. doi: $10.1021 / \mathrm{ma} 051266 \mathrm{z}$

[34] Tsuji, H., Sawada, M. and Bouapao, L. (2009) Biodegradable polyesters as crystallization-accelerating agents of poly(1-lactide). ACS Applied Materials \& Interfaces, 1,
1719-1730. doi:10.1021/am9002759

[35] Neuendorf, R.E., Saiz, E., Tomsia, A.P. and Ritchie, R.O. (2008) Adhesion between biodegradable polymers and hydroxyapatite: Relevance to synthetic bone-like materials and tissue engineering scaffolds. Acta Biomaterialia, 4, 1288-1296. doi:10.1016/j.actbio.2008.04.006

[36] Kontakis, G.M., Pagkalos, J.E., Tosounidis, T.I., Melissas, J. and Katonis, P. (2007) Bioabsorbable materials in orthopaedics. Acta Orthopaedica Belgica, 73, 159-169.

[37] Kang, S., Hsu, H.D., Smith, P.B., Leugers, M.A. and Yang, X. (2001) A spectroscopic analysis of poly(lactic acid) structure. Macromolecules, 34, 4542-4548. doi:10.1021/ma0016026

[38] Meaurio, W., Zuza, E., Roderiguez, L.R. and Saraszua, J.R. (2006) Conformational behavior of poly(L-lactide) studied by infrared spectroscopy. The Journal of Physical Chemistry, 110, 5790-5800. doi:10.1021/jp055203u

[39] Pan, P., Zhu, B., Dong, T., Yazawa, K., Shimizu, T., Tansho, M. and Inoue, Y. (2008) Conformational and microstructural characteristics of poly(L-lactide) during glass transition and physical aging. The Journal of Chemical Physics, 129, 184902-184912. doi:10.1063/1.3010368

[40] Yin, M. and Baker, G.L. (1999) Preparation and characterization of substituted polylactides. Macromolecules, 32, 7711-7718. doi:10.1021/ma9907183

[41] Simmons, T.L. and Baker, G.L. (2001) Poly(phenyllactide): Synthesis, characterization, and hydrolytic degradation. Biomacromolecules, 2, 658-663. doi:10.1021/bm005639+

[42] Trhnaille, T., Moller, M. and Gurny, R. (2004) Synthesis and ring-opening polymerization of new monoalkyl-substituted lactides. Journal of Polymer Science Part A: Polymer Chemistry, 42, 4379-4391. doi:10.1002/pola.20251

[43] Liu, T.Q., Simmons, T.L., Bohnsack, D.A., Mackay, M.E., Smith, M.R. and Baker G.L. (2007) Synthesis of polymandelide: A degradable polylactide derivative with polystyrene-like properties. Macromolecules, 40, 6040-6047. doi:10.1021/ma061839n

[44] Baker, G.L., Vogel, E.B. and Smith, M.R. (2008) Glass transitions in polylactides. Polymer Reviews, 48, 64-84. doi:10.1080/15583720701834208

[45] Yang, D., Qu, J., Li, B., Ng, F.F., Wang, X.C., Cheung, K.K., Wang, D.P. and Wu, Y.D. (1999) Novel turns and helices in peptides of chiral $\alpha$-aminoxy acids. Journal of the American Chemical Society, 121, 589-590. doi:10.1021/ja982528y

[46] Pullman, B. and Pullman, A. (1974) Molecular orbital calculations on the conformations of amino acid residues of proteins. Advances in Protein Chemistry, 128, 347-526. doi:10.1016/S0065-3233(08)60233-8

[47] Nandel, F.S., Malik, N., Singh, B and Jain, D.V.S. (1999) Conformational structure of peptides containing dehydroalanine: Formation of beta bend ribbon structure. International Journal of Quantum Chemistry, 72, 15-23. doi:10.1002/(SICI)1097-461X(1999)72:1<15::AID-QUA 2>3.0.CO;2-2

[48] Nandel, F.S., Malik, N., Singh, B. and Virdi, M. (1999) Designing of peptides with left handed helical structure by incorporating the unusual amino acids. Indian Journal of Biochemistry \& Biophysics, 36, 195-203.

[49] Lawrence, R.P. and Thompson, C.W.C. (1982) The boron analogue of glycine: A theoretical investigation of struc- 
ture and properties. Theochem, 5, 37-43. doi:10.1016/0166-1280(82)80105-X

[50] Aleman, C. and Casanovas, J. (1994) Ab initio SCF and force-field calculations on low-energy conformers of 2-acetylamino-2,N-dimethylpropanamide. Journal of the Chemical Society, Perkin Transactions, 2, 563-568. doi:10.1039/p29940000563

[51] Aleman, C. and Casanovas, J. (1995) Molecular conformational analyses of dehydroalanine analogues. Biopolymers, 36, 71-82. doi:10.1002/bip.360360107

[52] Weiner, S.J., Singh, U.C., O’Donell, T.J. and Kollman, P.A. (1984) Quantum and molecular mechanical studies on alanyl dipeptide. Journal of the American Chemical Society, 106, 6243-45. doi:10.1021/ja00333a021

[53] Mohle, K. and Hoffman, H.J. (1998) Secondary structure formation in N-substituted peptides. Journal of Peptide Research, 51, 19-28. doi:10.1111/j.1399-3011.1998.tb00412.x

[54] Adzubei, A.A., Eisenmenger, F., Tumanyan, V.G., Zinke, M., Brodzinski, S. and Esipova, N.G. (1987) Approaching a complete classification of protein secondary structure. Journal of Biomolecular Structure \& Dynamics, 5, 689-704.

[55] Adzubei, A.A and Sternberg, J.E. (1993) Left-handed polyproline II helices commonly occur in globular proteins. Journal of Molecular Biology, 229, 472-93. doi:10.1006/jmbi.1993.1047

[56] Van der Spoel, D., Lindahl, E., Hess, B., Groenhof, G., Mark, A.E. and Berendsen, H.J.C. (2005) GROMACS: Fast, flexible, and free. Journal of Computational Chemistry, 26, 1701-1718. doi:10.1002/jcc.20291

[57] Damm, W., Van Gunsteren, W. F. (2000) Reversible peptide folding: Dependence on molecular force field. Journal of Computational Chemistry, 21, 774-787. doi:10.1002/(SICI)1096-987X(20000715)21:9<774::AID -JCC6>3.3.CO;2-X

[58] Schuettelkopf, A.W. and Van Aalten, D.M.F. (2004) PRODRG-A tool for high-throughput crystallography of protein-ligand complexes. Acta Crystallographica, 60, $1355-1363$.

[59] Berendsen, H.J.C., Grigera, J.R. and Straatsma, T.P. (1987) The missing term in effective pair potentials. Journal of Physical Chemistry, 91, 6269-6271. doi:10.1021/j100308a038

[60] Berendsen, H.J.C., Postma, J.P.M., DiNola, A. and Haak, J.R. (1984) Molecular dynamics with coupling to an external bath. The Journal of Chemical Physics, 81, 36843690. doi:10.1063/1.448118

[61] Hess, B., Bekker, H., Berendsen, H.J.C. and Fraaije, JG.E.M. (1997) LINCS: A linear constraint solver for molecular simulations. Journal of Computational Chemistry, 18, 1463-1472.

doi:10.1002/(SICI)1096-987X(199709)18:12<1463::AID -JCC4>3.0.CO;2-H

[62] Essmann, U., Perera, L., Berkowitz, M.L., Darden, T., Lee, H. and Pedersen, L.G. (1995) A smooth particle mesh Ewald method. The Journal of Chemical Physics, 103, 8577-8592. doi:10.1063/1.470117

[63] Berendsen, H.J.C., Postma, J.P.M., Van Gunsteren, W.F. and Hermans, J. (1981) Interaction models for water in relation to protein hydration. In: Pullman, B., Ed., Intermolecular Forces, D. Reidel Publishing Company,
Dordrecht, 331-342.

[64] Brant, D.A., Tonelli, A.E. and Flory, P.J. (1969) The configurational statistics of random poly(lactic acid) chains. I. Experimental results. Macromolecules, 2, 225-227. doi:10.1021/ma60009a002

[65] Witzke, D.R. (1997) Introduction to properties, engineering and prospects of polylactide polymers. PhD Thesis, Michigan State University, East Lansing, 389.

[66] Tanimoto, S., Iwata, T., Yamaoka, H., Yamada, M. and Kobori, K. (2009) Conformational study of polypeptide chains grafted on the surface of polylactide latex particle. Research Leters in Material Sceince, 2009, 196950.

[67] Nandel, F.S. and Jaswal, R. (2007) New type of helix and 27 ribbon structure formation in poly $\Delta$ Leu peptides: Construction of a single-handed template. Biomacromolecules, 8, 3093-3101. doi:10.1021/bm700504h

[68] Nandel, F.S. and Kaur, H. (2003) Effect of terminal achiral and chiral residues on the conformational behaviour of poly $\Delta^{\mathrm{Z}} \mathrm{Phe}$ and analysis of various interactions. Indian Journal of Biochemistry \& Biophysics, 40, 265-73.

[69] Park, C. and Goddard III, W.A. (2000) Stabilization of $\alpha$-helices by dipole-dipole interactions within $\alpha$-helices. Journal of Physical Chemistry B, 104, 7784-7789. doi:10.1021/ip0001743

[70] Jing, F., Smith, M.R. and Backer, G.L. (2007) Cyclohexyl-substituted polyglycolides with high glass transition temperatures. Macromolecules, 40, 9304-9312. doi:10.1021/ma071430d

[71] Allen, F.H., Baalham, C.A., Lommerse, J.P.M. and Raithby, P.R. (1998) Carbonyl-carbonyl interactions can be competitive with hydrogen bonds. Acta Crystallographica Section B, 54, 320-329. doi:10.1107/S0108768198001463

[72] Allen, F.H., Dacies, J.E., Galloy, J.J., Johnson, O., Kennard, O., Macrae, C.F., Mitchell, E.M., Mitchell, G.F., Smith, J.M. and Watson, D.G. (1991) The development of version 3 and 4 of the Cambridge Structural Database System. Journal of Chemical Information and Modeling, 31, 187-204. doi:10.1021/ci00002a004

[73] Maccallum, P.H., Poet, R. and Milner-White, E.J. (1995) Coulombic interactions between partially charged mainchain atoms not hydrogen-bonded to each other influence the conformations of alpha-helices and anti-parallel betasheet. A new method for analyzing the forces between hydrogen bonding groups in proteins includes all the Coulombic interactions. Journal of Molecular Biology, 48, 361-373. doi:10.1016/S0022-2836(95)80056-5

[74] Maccallum, P.H., Poet, R. and Milner-White, E.J. (1995) Coulombic attractions between partially charged mainchain atoms stabilize the right-handed twist found in most beta-strands. Journal of Molecular Biology, 248, 374-384. doi:10.1006/jmbi.1995.0277

[75] Deane, C.M., Allen, F.H., Taylor, R. and Blundell, T.L. (2000) Carbonyl-carbonyl interactions stabilize the partially allowed Ramachandran conformations of asparagine and aspartic acid. Protein Engineering, 13, 1025-1028.

[76] Nandel, F.S. and Khare, B. (2005) Conformation of peptides constructed from achiral amino acid residues Aib and $\Delta^{Z}$ Phe: Computational study of the effect of L/DLeu at terminal positions. Biopolymers, 77, 63-73. doi:10.1002/bip.20128

[77] Nandel, F.S., Kaur, H., Malik, N., Shankar, N. and Jain, D.V.S. (2001) Conformational study of peptides contain- 
ing dehydrophenylalanine: Helical structures without hydrogen bonds. Indian Journal of Biochemistry \& Biophysics, 38, 417-425.

[78] Senes, A., Belandia, I.U. and Engelman, D.M. (2001) The $\mathrm{Ca}_{\alpha}-\mathrm{H}-\mathrm{O}$ hydrogen bond: A determinant of stability and specificity in transmembrane helix interactions. Proceedings of the National Academy of Sciences, 98, 9051-9056. doi:10.1073/pnas.161280798

[79] Steiner, T. (1997) Unrolling the hydrogen bond properties of $\mathrm{C}-\mathrm{H} \cdots \mathrm{O}$ interactions. Chemical Communications, $\mathbf{8}$,
727-734. doi:10.1039/a603049a

[80] Steiner, T. (1996) C-H-O hydrogen bonding in crystals. Crystallography Reviews, 6, 1-51. doi:10.1080/08893119608035394

[81] Palumbo, M., Cosani, A., Terbojevich, M. and Peggion, E. (1981) Conformational studies on synthetic polypeptides: poly (D-phenylglycine) and poly(D-cyclohexylglycine). International Journal of Biological Macromolecules, 3, 91-96. doi:10.1016/0141-8130(81)90073-8 